\title{
Once-punctured tori and knots in lens spaces
}

\author{
Kenneth L. BAKeR
}

\begin{abstract}
We determine the non-null homologous knots in lens spaces whose exteriors contain properly embedded once-punctured tori. All such knots arise as surgeries on the Whitehead link and are grid number 1 in their lens spaces. As a corollary, we classify once-punctured torus bundles that admit a lens space filling.
\end{abstract}

\section{Introduction}

Say a knot whose exterior contains a properly embedded once-punctured torus is an OPT-knot. In this article, we determine every non-null homologous OPT-knot $K$ in a lens space $X \not S^{1} \times S^{2}$ up to homeomorphism of the pair $(X, K)$. As a corollary, when combined with previous work of the author [1], we determine all once-punctured torus bundles that admit a lens space filling. See Theorem 1.1 and Corollary 1.3, respectively.

The lens space $L(r, q)$ is obtained by $-r / q$ Dehn surgery on the unknot in $S^{3}$ for coprime integers $r$ and $q$. Recall that $L(r, q) \cong L\left(r^{\prime}, q^{\prime}\right)$ if and only if $r^{\prime}= \pm r$ and $q^{\prime}= \pm q^{ \pm 1}$ modulo $r$.

Let $K$ be an OPT-knot in a lens space $X \neq S^{1} \times S^{2}$ and let $S$ be a oncepunctured torus properly embedded in its exterior $E(K)=X-N(K)$. Let $s$ be the minimal positive integer for which $s[K]=0$ in $H_{1}(X)$. We say $s$ is the order of $K$. Observe that $s$ is the number of times a meridian of $K$ minimally intersects $\partial S$ on $\partial E(K)$. Let $r$ be the order of $X \not S^{1} \times S^{2}$ so that $H_{1}(X) \cong \mathbb{Z} / r, X \cong L(r, q)$ for some $q$, and $0<s \leq r$.

Let $W$ denote the Whitehead link depicted on the left of figure 1. Let $W\left(\gamma_{1}, \gamma_{2}\right)$ denote Dehn surgeries of slopes $\gamma_{1}$ and $\gamma_{2}$ on the two components of $W$ in the standard coordinates. Let $Y$ denote the link of the "twisted" twochain with axis depicted on the middle and right of figure 1. Let $Y\left(\gamma_{1} ; \gamma_{2}, \gamma_{3}\right)$ denote Dehn surgeries of slope $\gamma_{1}$ on the axis and $\gamma_{2}$ and $\gamma_{3}$ on the other two components. Note the symmetries of $W$ and $Y$ which give $W\left(\gamma_{1}, \gamma_{2}\right)=$ $W\left(\gamma_{2}, \gamma_{1}\right)$ and $Y\left(\gamma_{1} ; \gamma_{2}, \gamma_{3}\right)=Y\left(\gamma_{1} ; \gamma_{3}, \gamma_{2}\right)$. We say a knot $K$ is unknotted if its exterior is a solid torus and is trivial if it is the boundary of an embedded disk. The type of a torus knot is defined in Section 3. 


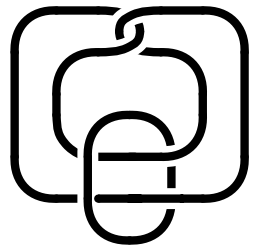

$W$

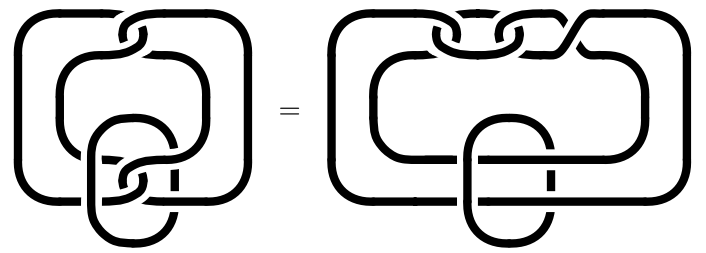

Y

Figure 1: The Whitehead link $W$ and the "twisted" two-chain with axis $Y$.

Theorem 1.1. If $K$ is a non-null homologous OPT-knot in a lens space $X \nsucceq S^{1} \times S^{2}$ then up to homeomorphism for some integers $k, \ell \neq 0$ either

(I) $K$ is the type $\{2,3\}$ torus knot in $L(6 k-1,2 k-1)$ arising as the core of the $-6+1 / k$ surgery in $W(-1,-6+1 / k)$,

(II) $K$ is the type $\{2,4\}$ torus knot in $L(8 k-2,2 k-1)$ arising as the core of the $-4+1 / k$ surgery in $W(-2,-4+1 / k)$,

(III) $K$ is the type $\{3,3\}$ torus knot in $L(9 k-3,3 k-2)$ arising as the core of the $-3+1 / k$ surgery in $W(-3,-3+1 / k)$,

(IV) $K$ is the knot in $L(9 k-3,3 k-2)$ arising as the core of the -3 surgery in $W(-3,-3+1 / k)$,

$(\mathrm{V}) K$ is the knot in $L(8 k \ell-2,4 k \ell-2 k-1)$ arising as the core of the -2 surgery in $Y(-2 ; 1 / k, 1 / \ell)$, or

(VI) $K$ is an unknotted knot in $L(r, q)$ arising as the core of the $-r / q$ surgery in $W(-r / q, \infty),|r| \neq 1$.

See figure 2. The once-punctured torus compresses in the exterior of $K$ if and only if $K$ belongs to family (VI).

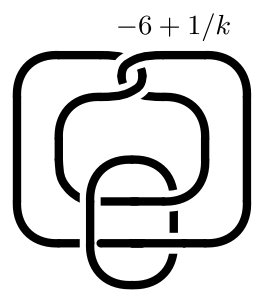

$-1$

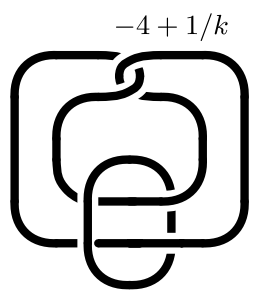

$-2$

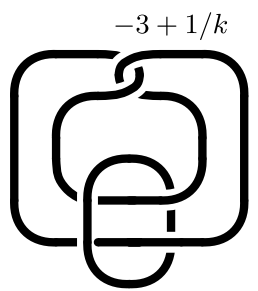

$-3$

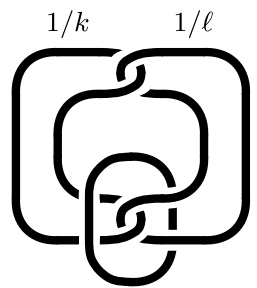

$-2$

Figure 2: Surgery descriptions of the five knotted families of non-null homologous OPT-knots in lens spaces. 
Remark 1.1. Table A.5 of [2] (with the Kirby calculus of Lemma 2.2) identifies $W(-2,-4+1 / k)$ as $L(8 k-2,4 k+1)$ but this is with an orientation reversing homeomorphism. The discrepancy may be noticed by setting $k=+1$ in families (II) and (III) above. That $W(-2,-4+1 / k)=$ $L(8 k-2,2 k-1)$ follows from Lemma 7.11.

Proof. If the exterior of $K$ is a solid torus, then it is the core of $-r / q$ Dehn surgery on the unknot in $S^{3}$ and belongs to family (VI). Now assume the exterior of $K$ is not a solid torus. When $K$ is a torus knot then by Lemma 3.1 it belongs to one of the first three families. When $K$ is not (necesarily) a torus knot then for $s \geq 3$ it follows from Theorem 6.1 that $K$ belongs to family (IV). For $s=2$, it follows from Theorem 7.1 that $K$ belongs to family (V).

Remark 1.2. The first three families of knots are all torus knots of orders $|-6 k+1|,|-4 k+1|$, and $|-3 k+1|$, respectively. The fourth and fifth families of knots are not torus knots (except for family (IV) with $k=1$ and for family (V) with $k=\ell= \pm 1$ ) and their orders are 3 and 2, respectively. Consequentially, only the knots in the first and last family represent generators of homology in their lens spaces.

Remark 1.3. Non-null homologous OPT-knots in $S^{1} \times S^{2}$ are knots that transversally intersect once a non-separating torus. For every non-separating embedding of a torus $T$ in $S^{1} \times S^{2}$, one may easily construct many OPTknots $K$ intersecting $T$ once so that surface $S=T-N(K)$ is incompressible in the exterior of $K$.

A (knotted) knot or link in a three-manifold has tunnel number 1 if the complement of an open neighborhood of some arc properly embedded in the exterior of the knot or link is a handlebody.

Corollary 1.1. Each non-null homologous OPT-knot in a lens space has tunnel number 1 .

Proof. Theorem 1.1 shows that each non-null homologous OPT-knot in a lens space arises from Dehn surgery on either the Whitehead link $W$, which itself has tunnel number 1 or the link $Y$. Those knots arising from surgery on $Y$ have exterior $Y(\cdot ; 1 / k, 1 / \ell)$ for which an unknotted properly embedded arc through disk bounded by one component of the chain and disjoint from the other may be seen to be an unknotting tunnel. This all follows more 
simply from Theorem 1.3 since grid number 1 knots are all 1-bridge with respect to the Heegaard torus and hence tunnel number 1.

Corollary 1.2. Let $k, \ell$ be non-zero integers. Up to homeomorphism of unoriented knot in a lens space: $L(6 k-1,2 k-1)$ contains exactly one knot-

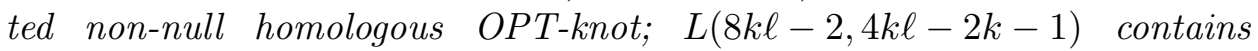
exactly two if $k= \pm 1$ or $\ell= \pm 1$, exactly three if $k=\ell= \pm 2$, and exactly one otherwise; $L(9 k-3,3 k-2)$ contains exactly three if $k=-3$ and exactly two otherwise. Only $L(30,11)$ contains three.

Proof. From Theorem 1.1 it is clear that these lens spaces contain at least as many as claimed. To show they contain no more, we must examine when these three families of lens spaces coincide. First observe that $2 \mid 8 k \ell-2$ and $3 \mid 9 k-3$ but $2 / 6 k-1$ and $3 / 6 k-1$; hence $L(6 k-1,2 k-1)$ contains no other non-null homologous OPT-knots.

Now notice that since $(3 j-2)^{2} \equiv 1$ modulo $9 j-3$, if $L(9 j-3,3 j-2) \cong$ $L(8 k \ell-2,4 k \ell-2 k-1)$ for some $j, k, \ell \in \mathbb{Z}$ then, from the classification of lens spaces, it must be the case that $(4 k \ell-2 k-1)^{2} \equiv 1$ modulo $8 k \ell-2$. One may show that this last congruence holds if and only if either $k=0$, $\ell=0$, or $k=\ell$. Since $9 j-3 \neq \pm 2$ for any integer $j$, we must have $k=\ell$. Because $\quad-L\left(8 k^{2}-2,4 k^{2}-2 k-1\right)=L\left(8(-k)^{2}-2,4(-k)^{2}-2(-k)-1\right)$, let us assume $k>0$. Then since $0<4 k^{2}-2 k-1<\frac{1}{2}\left(8 k^{2}-2\right)$, in order to have $L\left(8 k^{2}-2,4 k^{2}-2 k-1\right)= \pm L(9 j-3,3 j-2)$ one of the following occurs. If $j>0$ then $8 k^{2}-2=9 j-3$ and $4 k^{2}-2 k-1=3 j-2$ which implies $k=1$ and $j=1$. If $j<0$ then $8 k^{2}-2=-9 j+3$ and $4 k^{2}-2 k-1=$ $-3 j+2$, which implies $k=2$ and $j=-3$.

Therefore, $L(8 k \ell-2,4 k \ell-2 k-1)= \pm L(9 j-3,3 j-2)$ if either $k=$ $\ell= \pm 1$ and $j=1$ or $k=\ell= \pm 2$ and $j=-3$. Up to homeomorphism (taking $k=\ell>0)$, these are the lens spaces $L(6,1)$ and $L(30,11)$, respectively. In $L(6,1)=W(-2,-3)$ there are two homeomorphism types of knotted non-null homologous OPT-knots, they correspond to the cores of the surgeries in the surgery description, and both are torus knots. In $L(30,11)=$ $W(-3,-10 / 3)=Y(-2 ; 1 / 2,1 / 2)$ there are three homeomorphism types of knotted non-null homologous OPT-knots, they correspond to the cores of the $-10 / 3,-3$ and -2 -surgeries in the surgery description, and only the first is a torus knot.

\subsection{Lens space fillings of once-punctured torus bundles}

By a genus one fibered knot, or GOF-knot for short, we mean a null homologous knot in a closed orientable three-manifold whose exterior is a once- 
punctured torus bundle; a GOF-knot is the binding of an open book whose pages are once-punctured tori.

Burde and Zieschang use the homology of once-punctured torus bundles in their proof that a GOF-knot in $S^{3}$ is either a trefoil or has exterior homeomorphic to that of the figure 8, [3]. Gonzáles-Acuña pins this down concluding that the GOF-knots in $S^{3}$ are the trefoils and the figure 8, [4].

Passing from $S^{3}$ to lens spaces, Morimoto describes all the GOF-knots in a handful of lens spaces, [5]. In [1], we extend Morimoto's result by classifying all the GOF-knots in all lens spaces. Using the homology of once-punctured torus bundles in a manner similar to Burde and Zieschang, Baldwin observes that if a once-punctured torus bundle is the exterior of a non-null homologous knot in a lens space of prime order, then the once-punctured torus bundle is homeomorphic to the exterior of a trefoil, [6]. We now complete the classification of knots in lens spaces with once-punctured torus bundle exteriors in Theorem 1.2 and describe the monodromy of a once-punctured torus bundle admitting a lens space filling in Corollary 1.3.

Let $x$ and $y$ be two simple closed curves on $S$ that transversally intersect once; let $\tau_{x}$ and $\tau_{y}$ be right-handed Dehn twists along these curves. Monodromies of once-punctured torus bundles are considered up to isotopy.

Theorem 1.2. If $K$ is a non-null homologous knot in the lens space $X$ with a once-punctured torus bundle of monodromy $\phi$ as its exterior, then up to homeomorphism for some integer $k \neq 0$ either

(I) $K$ is a type $\{2,3\}$ torus knot in $L(6 k-1,2 k-1)$ with $\phi \cong \tau_{x} \tau_{y}$,

(II) $K$ is a type $\{2,4\}$ torus knot in $L(8 k-2,2 k-1)$ with $\phi \cong \tau_{x}^{2} \tau_{y}$,

(III) $K$ is a type $\{3,3\}$ torus knot in $L(9 k-3,3 k-2)$ with $\phi \cong \tau_{x}^{3} \tau_{y}$,

(IV) $K$ is the non-torus knot in $L(12,5)$ arising as the core of the -3 surgery in $W(-3,-4)$ with $\phi \cong \tau_{x}^{4} \tau_{y}$, or

(V) $K$ is the non-torus knot in $L(10,3)$ arising as the core of the -2 surgery in $W(-2,-5)$ with $\phi \cong \tau_{x}^{5} \tau_{y}$.

Proof. When $K$ is a torus knot families (I), (II) and (III) follow from Lemma 3.1. When $s \geq 3$ Theorem 6.1 and in particular Lemma 6.15 gives the knot in family (IV) with $k=-1$; the knot in family (IV) with $k=+1$ is a torus knot. When $s=2$ Theorem 7.1 and in particular Lemma 7.14 (with Lemma 7.10) gives the knot in family (V) with $k=-\ell= \pm 1$; the knot in family (V) with $k=\ell= \pm 1$ is a torus knot. 
Remark 1.4. In Theorem 1.2 the first three monodromies are periodic, the fourth is reducible, and the fifth is pseudo-Anosov (see e.g. [7]). Indeed the fourth exterior, $W(-4, \cdot)$, contains an essential Klein bottle. The fifth exterior, $W(-5, \cdot)$, is the exterior of figure 8 sister.

Corollary 1.3. Up to homeomorphism, a once-punctured torus bundle admits a lens space filling if and only if it has monodromy $\phi \cong \tau_{x}^{k} \tau_{y}^{2} \tau_{x}^{\ell} \tau_{y}^{-1}$ for $k, \ell \in \mathbb{Z}$.

Proof. Let $\Delta$ denote the distance of the slope of a lens space filling of a once-punctured torus bundle from the slope of the boundary of a fiber; see Section 2.1.

If $\Delta>1$, then the core of the filling is a knotted non-null homologous OPT-knot in a lens space with fibered exterior. Hence, $\phi$ must be as in Theorem 1.2. These are equivalent to choosing $k \in\{1,2,3,4,5\}$ and $\ell=0$ in the expression $\phi=\tau_{x}^{k} \tau_{y}^{2} \tau_{x}^{\ell} \tau_{y}^{-1}$.

If $\Delta=1$, then the corresponding knot is a (null homologous) genus one fibered knot. In [1] we show that $\phi$ has the stated form and that every such $\phi$ is the monodromy of a genus one fibered knot in a lens space (including $\left.S^{1} \times S^{2}\right)$.

If $\Delta=0$ then the filling is a torus bundle. But this cannot be a lens space.

Corollary 1.4. Every non-null homologous knot $K$ in a lens space with a once-punctured torus bundle exterior admits a non-trivial lens space surgery. In particular there is a GOF-knot in a lens space with the same exterior as $K$.

Proof. The exteriors of the five families of non-null homologous knots in lens spaces with once-punctured torus bundle exteriors listed in Theorem 1.2 are $W(-1, \cdot), W(-2, \cdot), W(-3, \cdot), W(-4, \cdot)$ and $W(-5, \cdot)$, respectively. The core of the $\infty$ filling of the remaining component of $W$ gives a GOF-knot in $L(1,1), L(2,1), L(3,1), L(4,1)$ and $L(5,1)$, respectively.

Remark 1.5. Each of these GOF-knots in lens spaces that admit nontrivial lens space surgeries (begetting the knots of Theorem 1.2) may be visualized up to homeomorphism as the plumbing of the +1 -Hopf band in $S^{3}$ onto the $r$-Hopf band in $L(r, 1)$ for $r=1,2,3,4,5$. We say an $r$-Hopf link is the two component fibered link in the lens space $L(r, 1)$ with an annulus fiber whose monodromy is $r$ Dehn twists along the core curve of the 
annulus; that is, the binding of an open book for $L(r, 1)$ with annular pages. An $r$-Hopf band is then an annulus fiber of an $r$-Hopf link. See [8].

\subsection{Grid number 1 knots}

Recently, the advent of combinatorial link Floer homology for links in $S^{3},[9]$ and [10], has brought to attention Cromwell's arc presentations of links, [11] and [12]. These may be reinterpreted as toroidal grid diagrams of links. One observes that analogous toroidal grid diagrams may be defined for knots in lens spaces too. Grigsby employs this in [13].

We define grid number 1 knots in Section 2.3 and discuss them here. Let us first note that in an oriented lens space the non-trivial elements of homology are canonically identified with the oriented grid number 1 knots. In various senses, these grid number 1 knots are "small" or "fundamental". For example, from the presentation of a knot $K$ as a grid number 1 knot one quickly obtains that the Heegaard Floer knot homology of $K$ is exceptionally simple with just one generator in each $\operatorname{Spin}^{c}$ structure. In fact, each grid number 1 knot is a "U-knot" as in Definition 5.2 of [14]. The relationship of a knot having grid number 1 and such particular simple Heegaard Floer homology is further examined in [15-17]. Indeed, the known knots in $S^{3}$ that admit integral Dehn surgeries yielding lens spaces, i.e., the classical Berge knots, all correspond to grid number 1 knots in the resulting lens spaces; see [18]. Yet another aspect of the simplicity of grid number 1 knots is demonstrated in the following theorem.

Theorem 1.3. Every non-null homologous OPT-knot in a lens space has grid number 1 .

Proof. Refer to Theorem 1.1 for the classification of non-null homologous OPT-knots in lens spaces. That the grid number of $K$ is 1 follows from Lemma 3.2 for the torus knots, from Lemma 6.11 when $s \geq 3$, from Lemma 7.9 when $s=2$, and trivially for the unknotted knots.

This suggests a question. First, let us say a properly embedded, connected, orientable surface $S$ in the exterior $E(K)=M-N(K)$ of a knot $K$ in some closed orientable three-manifold $M$ is a rational Seifert surface for $K$ if an orientation on $S$ causes $\partial S$ to be a coherently oriented collection of circles on $\partial E(K)$. Then the (rational) genus of $K$ is the minimal genus among all rational Seifert surfaces for $K$. 
Question 1.1. Do the grid number 1 knots in a lens space have minimal genus among knots in their homology classes?

Note that since the number of boundary components of a rational Seifert surface is invariant for knots within a homology class, this question may be rephrased in terms of Euler characteristics.

Further supporting the simplicity of grid number 1 knots in lens spaces, Ozsváth and Szabó algebraically prove that a grid number 1 knot in a lens space representing a generator of homology has fibered exterior, [19]. They show this using a theorem of Stallings that gives an algebraic characterization of fibered orientable three-manifolds, [20], together with a theorem of Brown about the finite generation of kernels of characters of two-generator one-relator groups, [21]. In accordance with this notion of simplicity, one might suspect that all grid number 1 knots have fibered exterior. Alas, this is not so.

Corollary 1.5. Not every grid number 1 knot has fibered exterior.

Proof. By Theorem 1.2, families (IV) and (V) of non-null homologous OPTknots are not fibered if $k \neq \pm 1$. This with Theorem 1.3 gives the result.

Because the OPT-knots in families (II), (III), (IV) and (V) do not represent generators of homology (their orders are strictly less than the orders of the lens spaces that contain them) the application of Brown's theorem in concert with Stallings' theorem does not work as cleanly. It is not uncommon, as in families (II) and (III) and in $L(p, 1)$, to find grid number 1 knots that are torus knots, and hence have fibered exterior, but do not represent generators of homology. Together Theorems 1.2 and 1.3 show that there are but two non-torus OPT-knots of grid number 1 whose exteriors are fibered and yet do not represent generators of homology.

Question 1.2. Aside from torus knots and ones that represent generators of homology, which grid number 1 knots have fibered exterior?

\section{Preliminaries}

Lemma 2.1. Let $S$ be a once-punctured torus properly embedded in the exterior of a non-null homologous knot $K$ in a lens space $X\left(¥ S^{1} \times S^{2}\right)$. If $S$ compresses then $K$ is an unknotted knot. 
Proof. Let $S^{\prime}$ be the disk resulting from a compression of $S$. Then $N(N(K) \cup$ $\left.S^{\prime}\right)$ is a punctured lens space of the same order as $K$. Since lens spaces are prime, $X-N\left(N(K) \cup S^{\prime}\right)$ is a 3-ball. Then $E(K)=X-N(K)$ is obtained by attaching a 1-handle that is a collar neighborhood of $S^{\prime}$ to this ball. Hence $E(K)$ is a solid torus.

Remark 2.1. If $X=S^{1} \times S^{2}$ then this lemma is false. Let $K$ be a knotted knot intersecting $S_{0}=\{0\} \times S^{2}$ once transversally. Then $K$ is non-null homologous and any tubing of $S_{0}-N(K)$ to itself in the exterior of $K$ along an arc whose ends approach the same side of $S_{0}$ forms a compressible once-punctured torus.

\subsection{Slopes and surgeries}

A slope is an isotopy class of essential simple closed curves on a torus. If $\gamma$ and $\delta$ are two slopes, then $\Delta(\gamma, \delta)$ denotes their distance, the minimal geometric intersection number among representatives of $\gamma$ and $\delta$ in the torus. If $c$ and $d$ are elements of $\gamma$ and $\delta$ respectively then we also write $\Delta(c, d)=\Delta(\gamma, \delta)$. For example, in this article we assume that for a meridian $\mu$ of $K, \Delta(\mu, \partial S)=s$ on $\partial N(K)$.

Given a link $K=K_{1} \cup \cdots \cup K_{n}$, let $K\left(\gamma_{1}, \ldots, \gamma_{n}\right)$ denote the manifold obtained by $\gamma_{i}$-Dehn surgery on each component $K_{i}$ for $i=1, \ldots, n$. We use the "slope" $\gamma_{i}=$. to indicate that a tubular neighborhood of $K_{i}$ is removed and left unfilled.

For a knot $K$ in $S^{3}$, we use standard meridian-longitude coordinates for its Dehn surgery slopes: Arbitrarily orient $K$ and let $\langle[\mu],[\lambda]\rangle$ be a basis for $H_{1}(\partial N(K) ; \mathbb{Z})$ where $\mu$ is an oriented meridian positively linking $K$ once and $\lambda$ is the slope of the Seifert surface oriented parallel to $K$. A slope $\gamma$ represented by an oriented essential simple closed curve $\bar{\gamma}$ on $\partial N(K)$ is given the coordinate $a / b \in \mathbb{Q} \cup\left\{\frac{1}{0}\right\}$ if $[\bar{\gamma}]=a[\mu]+b[\lambda]$ in $H_{1}(\partial N(K) ; \mathbb{Z})$.

Let $W$ denote the Whitehead link and $Y$ denote the twisted two-chain with axis shown in figure 1 . The pretzel link $N=P(-2,-2,-2)$ has exterior homeomorphic to the the so-called "magic" manifold, see Gordon and Wu $[22,23]$ and, for our purposes in particular, Martelli and Petronio [2].

Lemma 2.2. As shown in figure 3, $N(\alpha, \beta,+1)=W(\alpha-1, \beta-1)$, $Y(\alpha ; \beta,+1)=W(\alpha, \beta-4)$, and $Y(\alpha ; \beta,-1)=W\left(-\alpha^{\prime},-\beta-4\right)$. The last equality requires a mirror, and if $\alpha$ has slope $a / b$ with $a>0$, then $\alpha^{\prime}$ has slope $a /(b+a)$. 

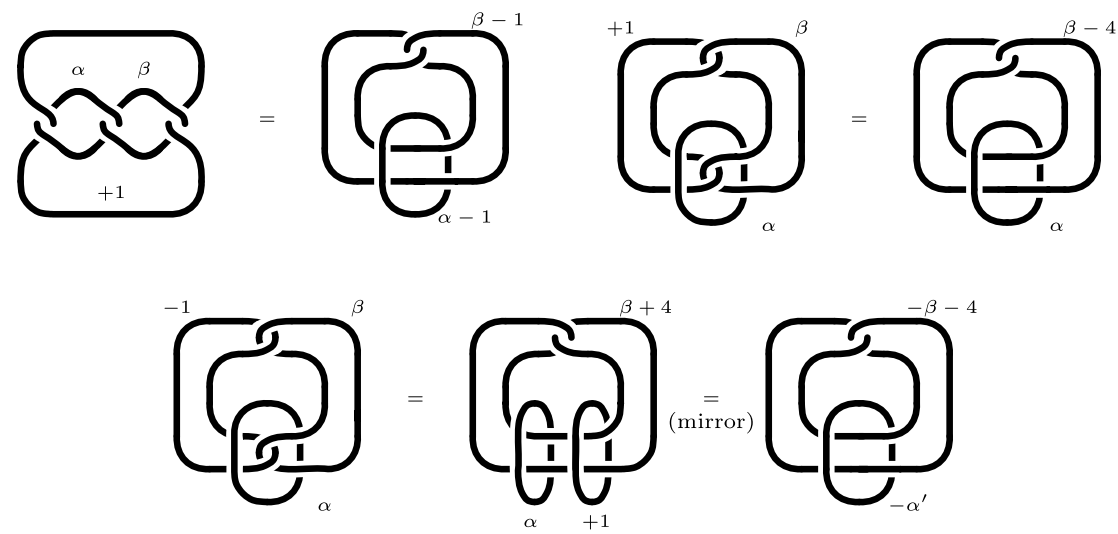

Figure 3: Kirby calculus gives the relationships $N(\alpha, \beta,+1)=W(\alpha-1$, $\beta-1), \quad Y(\alpha ; \beta,+1)=W(\alpha, \beta-4)$, and, with mirroring, $Y(\alpha ; \beta,-1)=$ $W\left(-\alpha^{\prime},-\beta-4\right)$.

In particular, we have $Y(-2 ; \beta,+1)=W(-2, \beta-4)$ and, with mirroring, $Y(-2 ; \beta,-1)=W(-2,-\beta-4)$.

Proof. These are straightforward applications of Kirby calculus. See chapters 4 and 5 of [24], for example.

Lemma 2.3 (Martelli-Petronio, [2]). $W(\alpha, \beta)$ is a lens space if and only if $\{\alpha, \beta\}$ is $\{-1,-6+1 / k\},\{-2,-4+1 / k\},\{-3,-3+1 / k\}$, or $\{p / q, \infty\}$ for some $k, p, q \in \mathbb{Z}$ and $(p, q)=1$.

Proof. Using Lemma 2.2 this is a direct consequence of the results of Martelli and Petronio listed in table A.5 of [2].

Lemma 2.4 (Proposition 3, [25]). $W(p / q, \cdot)$ fibers over the circle if and only if $|q| \leq 1$. For each $p \in \mathbb{Z}, W( \pm p / 1, \cdot)$ fibers with a once-punctured torus fiber and monodromy $\phi \cong \tau_{x}^{\mp p} \tau_{y}$.

Remark 2.2. If $q=0$ then $W(p / q, \cdot)$ is a solid torus and fibers over the circle with a disk fiber. For $q \neq 0$, we refer the reader to [25] for a proof. Let us mention, nevertheless, that the monodromy is most directly viewed by regarding $W$ as the result of -1 surgery on one component of the Borromean rings (contrast with the link $Y$ ). Then $W( \pm p, \cdot)$ may be seen as the pair of surgeries $\pm p$ and -1 on the untwisted two-chain link in the solid torus. A representative once-punctured torus fiber is a meridional disk tubed once to 
be disjoint from the chain link. Pushing the tube through the surgery solid torus of each of these link components effects a Dehn twist.

Lemma 2.5. $Y(\cdot ; 1 / k, 1 / \ell)$ fibers over the circle with a OPT fiber if and only if $|k|=|\ell|=1$. The manifold is a solid torus if and only if $k=0$ or $\ell=0$.

Proof. In the link $Y$, the axis of the two-chain bounds a disk that intersects a single component of the two-chain twice and is disjoint from the other component. Tube this disk along the first component to form a OPT $S$ disjoint from the two-chain and incompressible in the exterior of $Y$. Since trivial surgery on one component of the two-chain leaves the other component as a trivial knot, $Y(\cdot ; 1 / k, 1 / \ell)$ is a solid torus if $k$ or $\ell$ is 0 . That the manifold $Y(\cdot ; 1 / k, 1 / \ell)$ is a solid torus only if $k$ or $\ell$ is 0 follows from a homology calculation (as well as the Alexander polynomial calculation below).

If, say, $|\ell|=1$, then by Lemma $2.2 Y(\cdot ; 1 / k, \pm 1) \cong W(\cdot,-4 \pm 1 / k)$. By Lemma 2.4 this fibers (with fiber $S$ ) if and only if $|k|=1$. By symmetry, a similar argument works if $|k|=1$.

Viewing $Y(\cdot ; 1 / k, 1 / \ell)$ as surgery on the two-chain in the solid torus complement of the axis of $Y$, we may compute its Alexander polynomial to be $\Delta(t)=k \ell\left(t+2+t^{-1}\right)-1$. (The first homology of the infinite cyclic cover of $Y(\cdot ; 1 / k, 1 / \ell)$ minus the lifts of the chain link is generated as a $\mathbb{Z}\left[t^{ \pm 1}\right]$ module by $\alpha$ and $\beta$. The lifts of surgery on the chain link gives relations
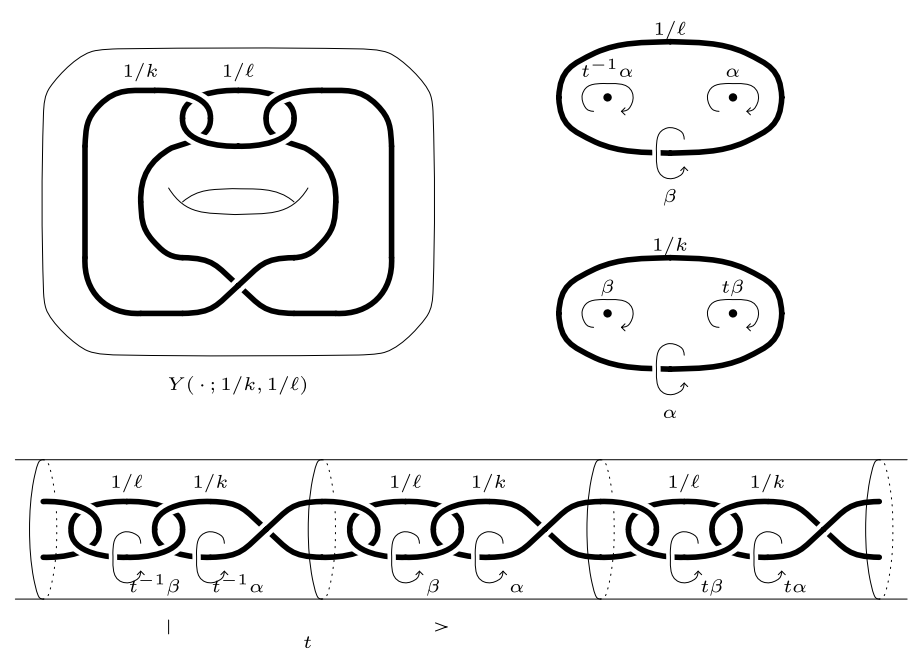

Figure 4: The Alexander polynomial of $Y(\cdot, 1 / k, 1 / \ell)$ may be computed from its surgery description on a two-chain link in a solid torus. 
$\ell\left(\alpha+t^{-1} \alpha\right)=\beta$ and $k(t \beta+\beta)=\alpha$ from which we obtain the Alexander polynomial. See figure 4.) Since the polynomial is monic only if $|k \ell| \leq 1$, $Y(\cdot ; 1 / k, 1 / \ell)$ cannot be fibered unless $|k \ell| \leq 1$.

\subsection{Heegaard splittings and height functions}

A lens space $X$ has a unique genus one Heegaard splitting: there is a torus, unique up to isotopy, that divides $X$ into two solid tori, e.g. [26]. These are referred to as the Heegaard torus and Heegaard solid tori accordingly. Endow $X$ with a height function $X \rightarrow[-\infty,+\infty]$ where the preimages of heights in $(-\infty,+\infty)$ are Heegaard tori and the preimages of $\pm \infty$ are the two unknotted circles. Let $\hat{T}$ be a the preimage of 0 . We refer to the Heegaard solid tori bounded by $\hat{T}$ and the objects in them as being above or below $\hat{T}$ depending on their relationship with respect to the height function on $X$. The colors amber and blue will also be used, respectively. Denote the amber and blue Heegaard solid tori as $V_{a}$ and $V_{b}$. Let $K_{a}=K \cap V_{a}$ and $K_{b}=K \cap V_{b}$. Let $m_{a}$ and $m_{b}$ be meridians of $V_{a}$ and $V_{b}$, respectively.

\subsection{Grid number 1 knots}

Assume the meridians $m_{a}$ and $m_{b}$ of $V_{a}$ and $V_{b}$ respectively intersect minimally on $\hat{T}$. Therefore in $L(r, q) \neq S^{3}, S^{1} \times S^{2}$ they intersect in $r>2$ points. The (unoriented) grid number 1 knots are defined by unordered pairs of distinct points $\{z, w\}$ of $m_{a} \cap m_{b}$. Let $D_{a}$ and $D_{b}$ be meridional disks of $V_{a}$ and $V_{b}$ with boundaries $m_{a}$ and $m_{b}$, respectively. Given a pair of distinct points $\{z, w\}$ of $m_{a} \cap m_{b}$ there is a properly embedded arc $K_{a}$ and $K_{b}$ in $D_{a}$ and $D_{b}$, respectively, with these points as their endpoints. The knot $K=K_{a} \cup K_{b}$ is then a grid number 1 knot.

If in some direction along $m_{b}$ the two points $\{z, w\}$ are $n$ apart (i.e., there are $n-1$ points of $m_{a} \cap m_{b}$ in the interior of an arc of $m_{b}$ between $z$ and $w)$ then we say $K$ is the $n$th grid number 1 knot in $L(r, q)$ along $m_{b}$. Taken along $m_{a}, K$ is the $n q$ th grid number 1 knot where $n q$ is reduced modulo $r$. Because we are working with knots in lens spaces up to homeomorphism we will understand that saying $K$ is the $n$th grid number 1 knot means the count of $n$ is taken with respect to either $m_{a}$ or $m_{b}$. In this article, for the torus knots at hand, the geometry will make apparent the correct choice; for the non-torus knots at hand, the symmetry makes the choice irrelevant. 


\section{Torus knots}

A torus knot $K$ in a lens space is an essential simple closed curve on $\hat{T}$. The exterior of a torus knot is a Seifert fiber space over the disk with (at most) two exceptional fibers and is fibered over $S^{1}$ unless the curve is isotopic to a meridian of one of the Heegaard solid tori. In this latter situation, the curve bounds a disk in the lens space and is hence the trivial knot; its exterior is the connect sum of the lens space and a solid torus.

If $K$ is a torus knot in the lens space $X=V_{a} \cup_{\hat{T}} V_{b}$, then let $\delta_{a}=$ $\Delta\left(K, m_{a}\right)$ and $\delta_{b}=\Delta\left(K, m_{b}\right)$ viewing the curves on the Heegaard torus. Then we say that $K$ is a type $\left\{\delta_{a}, \delta_{b}\right\}$ torus knot. The type of a torus knot reveals its element of homology with respect to the two canonical generators, the unknotted cores of $V_{a}$ and $V_{b}$.

Lemma 3.1. If $K$ is a non-null homologous torus knot and an OPT-knot then either $K$ is unknotted or $E(K)$ fibers over the circle with a oncepunctured torus fiber and, up to homeomorphism, for some integer $k \neq 0$, either

(I) $K$ is a type $\{2,3\}$ torus knot in $L(6 k-1,2 k-1)$ arising as the core of the $-6+1 / k$-surgery in $W(-1,-6+1 / k)$ with monodromy $\phi \cong \tau_{x} \tau_{y}$,

(II) $K$ is a type $\{2,4\}$ torus knot in $L(8 k-2,2 k-1)$ arising as the core of the $-4+1 / k$-surgery in $W(-2,-4+1 / k)$ with monodromy $\phi \cong$ $\tau_{x}^{2} \tau_{y}$, or

(III) $K$ is a type $\{3,3\}$ torus knot in $L(9 k-3,3 k-2)$ arising as the core of the $-3+1 / k$-surgery in $W(-3,-3+1 / k)$ with monodromy $\phi \cong \tau_{x}^{3} \tau_{y}$.

Proof. Since the exterior of a non-trivial (and hence non-null homologous) torus knot is fibered, if it is an OPT-knot with a compressible properly embedded once-puncutred torus in its exterior, then Lemma 2.1 says that the knot is unknotted. Therefore let us now assume the once-punctured torus in the exterior of $K$ is incompressible.

The exterior $E(K)$ of a torus knot $K$ is the union of two solid tori $V_{a}^{\prime}$ and $V_{b}^{\prime}$ along essential annuli in their boundary. (View $V_{a}$ and $V_{b}$ as the Heegaard solid tori above and below the Heegaard torus on which $K$ lies. Dig out a trench from each along $K$ to form $V_{a}^{\prime}$ and $V_{b}^{\prime}$.) Let $A=V_{a}^{\prime} \cap V_{b}^{\prime}$ be this annulus, and let $D_{i}$ be meridional disks of $V_{i}^{\prime}$ minimally intersecting $A, i=a, b$. It may be arranged so that $D_{a}$ and $D_{b}$ are disjoint. Set $\delta_{i}=$ $\left|\partial D_{i} \cap A\right|$ and let $\lambda=\operatorname{lcm}\left(\delta_{a}, \delta_{b}\right)$. We may now easily view $E(K)$ as a fiber bundle over the circle with fiber $S$ composed of $\lambda / \delta_{1}$ parallel copies of $D_{a}$ 
in $V_{a}^{\prime}$ joined to $\lambda / \delta_{b}$ parallel copies of $D_{b}$ in $V_{b}^{\prime}$ along $\lambda$ arcs in $A$. In order for $S$ to be a once-punctured torus, we must have $\chi(S)=\lambda / \delta_{a}+\lambda / \delta_{b}-\lambda=$ -1 . Rewritten as $1 / \delta_{a}+1 / \delta_{b}+1 / \lambda=1$, the solutions are clear. The only positive integer solutions are $\left\{\delta_{a}, \delta_{b}\right\}=\{2,3\}$ and $\left\{\delta_{a}, \delta_{b}\right\}=\{2,4\}$ if $\delta_{a} \neq \delta_{b}$ and $\delta_{a}=\delta_{b}=3$ otherwise. Therefore, the only possible torus knot types of OPT-knots are $\{2,3\},\{2,4\}$ and $\{3,3\}$. We now describe all such torus knots and their monodromies. To facilitate this, regard the disks $D_{i}$ as regular ideal $\delta_{i}$-gons.

Choosing $\delta_{a}=2$ and $\delta_{b}=3$, the fiber $S$ is assembled from three copies of $D_{a}$ joined to two copies of $D_{b}$ along 6 arcs. Sliding $S$ around $V_{a}^{\prime} \cup V_{b}^{\prime}$ through its monodromy back to itself permutes the three copies of $D_{a}$ around $V_{a}^{\prime}$ and the two copies of $D_{b}$ around $V_{b}^{\prime}$. Doing so three times returns each copy of $D_{a}$ to itself with a $\pi$ rotation. Doing so twice returns each copy of $D_{b}$ to itself with a $2 \pi / 3$ rotation. Hence the monodromy may be now visualized as a $\pi / 3$ rotation of a regular hexagon minus an open disk at the center with opposite sides identified; $\phi \cong \tau_{x} \tau_{y}$. See figure $5(\mathrm{I})$. Hence by Lemma $2.4 E(K) \cong W(-1, \cdot)$. Martelli and Petronio then show in table A.5 of [2] that $W(-1,-6+1 / k)=L(6 k-1,2 k-1)$ for $k \in \mathbb{Z}$ are the only lens space fillings of $W(-1, \cdot)$.

Choosing $\delta_{a}=2$ and $\delta_{b}=4$, the fiber $S$ is assembled from two copies of $D_{a}$ joined to one copy of $D_{b}$ along 4 arcs. The monodromy permutes the two copies of $D_{a}$. Done twice, each copy of $D_{a}$ returns to itself with a $\pi$ rotation. Done once, $D_{b}$ returns to itself with a $\pi / 2$ rotation. Hence the monodromy may be visualized as a $\pi / 2$ rotation of a square minus an open disk at the center with opposite sides identified; $\phi \cong \tau_{x} \tau_{y} \tau_{x} \cong \tau_{x}^{2} \tau_{y}$. See figure 5(II). Hence by Lemma $2.4 E(K) \cong W(-2, \cdot)$. Martelli and Petronio then show in table A.5 of [2] that $W(-2,-4+1 / k)=L(8 k-2,2 k-1)$ for $k \in \mathbb{Z}$ (see Remark 1.1) are the only lens space fillings of $W(-2, \cdot)$.

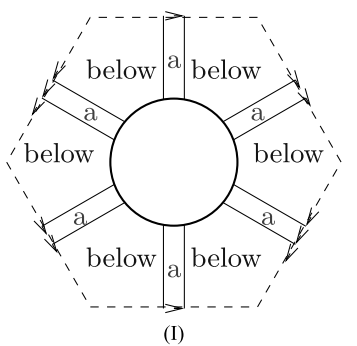

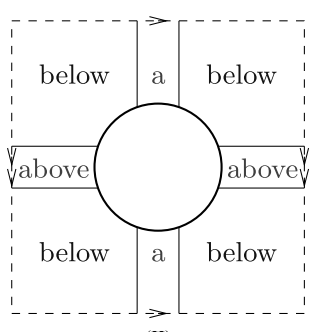

(II)

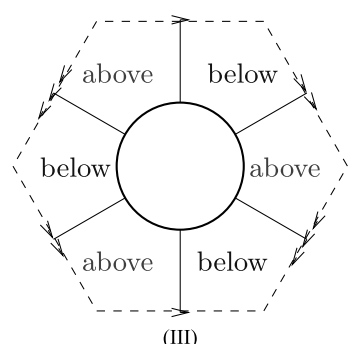

(III)

Figure 5: The fibers of torus knots. 
Choosing $\delta_{a}=3$ and $\delta_{b}=3$, the fiber $S$ is assembled from one copy of $D_{a}$ joined to one copy of $D_{b}$ joined along 3 arcs. The monodromy rotates each of the two disks by $2 \pi / 3$ (so that $|\partial S|=1$ ). Hence the monodromy may be visualized as a $2 \pi / 3$ rotation of a regular hexagon minus an open disk at the center with opposite sides identified; $\phi \cong \tau_{y} \tau_{x} \tau_{y} \tau_{x} \cong \tau_{x}^{3} \tau_{y}$. See figure 5(III). Hence by Lemma $2.4 E(K) \cong W(-3, \cdot)$. Martelli and Petronio then show in table A.5 of [2] that for each $W(-3,-3+1 / k)=L(9 k-3,3 k-2)$ for $k \in \mathbb{Z}$ are the only lens space fillings of $W(-3, \cdot)$.

Remark 3.1. One may notice that the lens spaces $L(9 k, 3 k+1)$ for $k \in \mathbb{Z}$ also contain type $\{3,3\}$ torus knots. The fibers for these knots however are thrice-punctured spheres.

Lemma 3.2. If $K$ is a knotted non-null homologous type $\left\{\delta_{a}, \delta_{b}\right\}$ torus knot and an OPT-knot then $K$ has grid number 1 and is the $\delta_{a}$ th or $\delta_{b}$ th grid number 1 knot.

Proof. Assume $K$ is a type $\left\{\delta_{a}, \delta_{b}\right\}$ torus knot. Let $m_{a}$ and $m_{b}$ be meridians of $V_{a}$ and $V_{b}$ that intersect minimally. Then $m_{a} \cup m_{b}$ forms a toroidal grid. Beginning at a vertex of the grid, travel along $m_{b}$ in one direction until the $\delta_{a}$ th vertex (intersection of $m_{a}$ ) is reached. Since $K$ is a type $\left\{\delta_{a}, \delta_{b}\right\}$ torus knot, for one of the choices of directions along $m_{b}$, the original vertex must now be $\delta_{b}$ vertices (intersections of $m_{b}$ ) away in one direction along $m_{a}$. Traveling in this direction back to the original vertex now completes a loop on $\hat{T}$ that is at least homotopic to $K$. If the interior of the part of this loop that runs along $m_{a}$ is slightly lifted up into $V_{a}$ and the interior of the part that runs along $m_{b}$ is slightly pushed down into $V_{b}$, then the knot created is the $\delta_{a}$ th grid number $1 \mathrm{knot}$, counted along $m_{b}$. Counting along $m_{a}$, this would be the $\delta_{b}$ th grid number 1 knot.

To show that this loop is actually simple and hence the torus knot we desire, we may simply show that no vertex of the grid in the loop is visited more than once. Number the intersections of $m_{a}$ and $m_{b}$ from 0 to $r-1$ in order along, say, $m_{b}$. Then, since $X=L(r, q)$, the intersection points appear in order along $m_{a}$ as $0, q, 2 q, \ldots,(r-1) q$ reduced modulo $r$. Therefore the loop on the grid encounters the following vertices in the order:

$$
0,1, \ldots, \delta_{a}, \delta_{a}+\dot{q}, \delta_{a}+2 \dot{q}, \ldots, \delta_{a}+\delta_{b} \dot{q}
$$

reduced modulo $r$ for some choice of $\dot{q}= \pm q^{ \pm 1}$. 
Lemma 3.1 gives three families for which we must check that such a sequence exists with no repetitions (except at the beginning and end). We do so to complete the proof.

(I) $k>0$ : The type $\{2,3\}$ torus knot in $L(6 k-1,2 k-1)$ gives the sequence

$$
0,1,2,2 k+1,4 k, 6 k-1
$$

$k<0$ : Set $k^{\prime}=-k$. The type $\{2,3\}$ torus knot in $L\left(6 k^{\prime}+1,2 k^{\prime}+1\right) \cong$ $L\left(6 k^{\prime}+1,4 k^{\prime}\right)$ gives the sequence

$$
0,1,2,4 k^{\prime}+2,2 k^{\prime}+1,6 k^{\prime}+1
$$

(II) $k>0$ : The type $\{2,4\}$ torus knot in $L(8 k-2,2 k-1)$ gives the sequence

$$
0,1,2,2 k+1,4 k, 6 k-1,8 k-2 \text {. }
$$

$k<0$ : Set $k^{\prime}=-k$. The type $\{2,4\}$ torus knot in $L\left(8 k^{\prime}+2,2 k^{\prime}+1\right) \cong$ $L\left(8 k^{\prime}+2,6 k^{\prime}+1\right)$ gives the sequence

$$
0,1,2,6 k^{\prime}+3,4 k^{\prime}+2,2 k^{\prime}+1,8 k^{\prime}+2
$$

(III) $k>0$ : The type $\{3,3\}$ torus knot in $L(9 k-3,3 k-2)$ gives the sequence

$$
0,1,2,3,3 k+1,6 k-1,9 k-3 \text {. }
$$

$k<0$ : Set $k^{\prime}=-k$. The type $\{3,3\}$ torus knot in $L\left(9 k^{\prime}+3,3 k^{\prime}+2\right) \cong$ $L\left(9 k^{\prime}+3,6 k^{\prime}+1\right)$ gives the sequence

$$
0,1,2,3,6 k^{\prime}+4,3 k^{\prime}+2,9 k^{\prime}+3
$$

Remark 3.2. If $K$ is unknotted in $L(r, q)$, then it may be identified as either the 1st or the $q$ th grid number 1 knot in $L(r, q)$.

\section{Fat-vertexed graphs of intersection}

Here, we set up machinery used in the remainder of this article. Litherland [27] first introduced fat-vertexed graphs into the study of intersecting surfaces. See Gabai [28], Rieck [29] and Gordon's survey [30] for the notion of thin position for knots in closed oriented three-manifolds and its interplay with intersecting surfaces. Our particular situation takes lead from Goda and 
Teragaito [31] where they examine the intersections between a (punctured) Heegaard torus and a Seifert surface in a knot exterior.

Recall our discussion in Section 2.2 about Heegaard splittings and height functions. Put $K$ in thin position with respect to the height function on our lens space $X$. Arrange that $\hat{T}$ is a thick torus with respect to $K$, which $K$ intersects, say, $t$ times. Let $H(K)$ be the closure of the tubular neighborhood $N(K)$. Denote $T=\hat{T}-N(K)=\hat{T} \cap E(K)$. Isotop $S$ rel- $\partial$ in $E(K)$ fixing $T$ to minimize $|S \cap T|$ among the positions of $S$ transversally intersecting $T$ in a collection of simple closed curves and simple proper arcs. Abstractly cap off $S$ with a disk to form the torus $\hat{S}$. Form the fat-vertexed graphs $G_{S}$ on $\hat{S}$ and $G_{T}$ on $\hat{T}$ as follows. We will view our diagrams of $G_{T}$ from above.

The fat-vertex of $G_{S}$ is the disk abstractly capping off $S$. The fat-vertices of $G_{T}$ are the disks $\hat{T} \cap H(K)$, the closures of the components of $\hat{T} \cap N(K)$. We find it useful to regard the fat-vertex of $G_{S}$ as just $\partial S$ and the fat-vertices of $G_{T}$ as meridional disks of $H(K)$.

The edges of $G_{S}$ and $G_{T}$ are the arcs of $S \cap T$. Typically we do not include the closed curve components of $S \cap T$ in our graphs, but we will occasionally bring them into play. The faces of $G_{S}$ and $G_{T}$ are the path closures of the components of the complements of the edges in $S$ and $T$ respectively. Here too one may occasionally care to include the simple closed curves of $S \cap T$ with the edges in the formation of the faces. A corner of a face $f$ is a component of $\partial f \cap \partial H(K)$. An $n$-gon is a disk face with $n$ edges and $n$ corners. For $n=1,2,3$, and 4 we call an $n$-gon a monogon, bigon, trigon, and tetragon respectively. By the minimality assumption on $|S \cap T|$, if $K$ is not a torus knot, then we may assume no face of $G_{S}$ or $G_{T}$ is a monogon (see e.g. Gordon [30]).

Number the intersections of $K$ with $\hat{T}$ from 1 to $t$ (taken modulo $t$ ) in order around $K$; the fat-vertices of $G_{T}$ are then labeled correspondingly. Around $\partial S$ each intersection with $\partial T$ inherits one of these labels. Observe that $\partial S$ intersects each component of $\partial T$ a total of $s$ times. Consequentially, the labels $\{1,2, \ldots, t\}$ appear in order $s$ times around $\partial S$. Therefore $G_{S}$ and $G_{T}$ have $s t / 2$ edges.

Each edge $e$ of $G_{T}$ connecting vertices $i$ and $j$ is also an edge $e$ of $G_{S}$ with end points labeled $i$ and $j$. We say $\{i, j\}$ is the label pair of the edge $e$ (as viewed in $G_{S}$ ). Since $G_{S}$ has just one vertex, $\hat{T}$ is separating, and both $S$ and $T$ are orientable, the two labels of a label pair for an edge in $G_{S}$ must have opposite parity; all edges on $G_{T}$ connect vertices of opposite parity. This is the Parity Rule; see [32], specialized to our context. (Since $G_{S}$ has just one vertex we need not label it. Edges in $G_{T}$ would all have the same label pair. Edges in $G_{S}$ connect its single vertex to itself.) 
Color each face $f$ of $G_{S}$ amber or blue according to whether a small collar neighborhood of $\partial f$ in $f$ lies above or below $\hat{T}$, respectively. This gives a "checkerboard" coloring of $G_{S}$. We may also choose to include the simple closed curves of $S \cap T$ on $G_{S}$ and then color the resulting faces according to the color of the Heegaard solid torus in which their interiors lie.

Let $H_{i, i+1}$ be the one-handle of $H(K)$ running from vertex $i$ to vertex $i+1$ and $K_{i, i+1}=K \cap H_{i, i+1}$. We say each $H_{i, i+1}$ and $K_{i, i+1}$ is unknotted if $K_{i, i+1}$ is contained in a meridional disk of the Heegaard solid torus in which it lies. Denote $\partial H_{i, i+1} \cap \partial H(K)$ by $\partial_{v} H_{i, i+1}$. We will always assume that $H_{1,2}$ is amber. Let $H_{a}=H(K) \cap V_{a}$ and $H_{b}=H(K) \cap V_{b}$.

A set of edges $\sigma=\left\{e_{1}, e_{2}, \ldots, e_{p}\right\}$ in $G_{S}$ each with the same label pair that together bound a single disk face of $G_{S}$ is called a Scharlemann cycle of length $p$. The label pair of $\sigma$ is the label pair of the edges of $\sigma$.

For any set of edges $\sigma$, if the subgraph of $G_{T}$ consisting of the edges of $\sigma$ and the vertices to which these edges are incident is contained in a disk on $\hat{T}$, then we say the edges of $\sigma$ lie in a disk. If the subgraph is contained in an annulus on $\hat{T}$ but not in a disk, then we say the edges of $\sigma$ lie in an essential annulus.

We say two edges of $G_{S}, G_{T}$ are parallel if they cobound a disk formed by a union of bigons (a "stack" of bigons) in $S, T$, respectively. We say two faces $f_{0}$ and $f_{1}$ of $G_{S}$ are parallel if there is an isotopy of $f_{0}$ to $f_{1}$ in $E(K)$ keeping $\partial f_{0} \cap T$ on $T$ and $\partial f_{0} \cap \partial H(K)$ on $\partial H(K)$; in particular if Int $\left(f_{0} \cup f_{1}\right) \cap T=\emptyset$ then $f_{0}$ and $f_{1}$ cobound a product region in $E(K)$.

\section{Scharlemann cycles of length 2 and 3}

Lemma 5.1 (Lemma 2.1, [31]). Let $\sigma$ be a Scharlemann cycle in $G_{S}$ of length $p$ with label pair $\{i, i+1\}$ where $p$ is 2 or 3 . Let $f$ be the face of $G_{S}$ bounded by $\sigma$. If the edges of $\sigma$ do not lie in a disk in $\hat{T}$ then they lie in an essential annulus $A$ in $\hat{T}$. Furthermore, if Int $f \cap \hat{T}=\emptyset$, then $M=$ $N\left(A \cup H_{i, i+1} \cup f\right)$ is a solid torus such that the core of $A$ runs $p$ times in the longitudinal direction of $M$.

Lemma 5.2. Continuing Lemma 5.1 in the case that Int $f \cap \hat{T}=\emptyset$, let $V$ be the solid torus bounded by $\hat{T}$ that contains $f$. Then the core of $A$ runs $p$ times in the longitudinal direction of $V$. In particular, the meridian of $V$ and the core of $A$ have distance $p$ on $\hat{T}$.

Proof. Since $A \cup H_{i, i+1} \cup f \subset V$ and $\left(A \cup H_{i, i+1} \cup f\right) \cap \hat{T}=A$, we may regard $M$ as its restriction to $V$ with $M \cap \hat{T}=A$. Because the annulus 
$\partial M-\operatorname{Int} A$ in $V$ is not parallel to $A$ through $M$, it must be parallel to $\hat{T}-$ Int $A$ through the solid torus $\overline{V-M}$. Therefore a meridional disk of $M$ extends to a meridional disk of $V$. Thus, the core of $A$ will run $p$ times in the longitudinal direction of $V$ as well. Hence, the meridian of $V$ and the core of the annulus $A$ have distance $p$ on $\hat{T}$.

Lemma 5.3. Let $\sigma_{i}$ and $\sigma_{j}$ be two Scharlemann cycles of length 2 in $G_{S}$ with disjoint label pairs $\{i, i+1\}$ and $\{j, j+1\}$, respectively. If the edges of each $\sigma_{i}$ and $\sigma_{j}$ lie in an essential annulus on $\hat{T}$, then $\sigma_{i}$ and $\sigma_{j}$ lie on opposite sides of $\hat{T}$.

Proof. Let $f_{k}$ be the face of $G_{S}$ bounded by $\sigma_{k}, k=i, j$. Assume $f_{i}$ and $f_{j}$ have the same color, say blue. Extend $f_{i}$ and $f_{j}$ across its corners radially to the cores of $H_{i, i+1}$ and $H_{j, j+1}$ to form the blue Möbius bands $g_{i}$ and $g_{j}$. Since the edges of $f_{k}$ lie in an essential annulus on $\hat{T}$, so does $\partial g_{k}$. By disk exchanges, eliminate all simple closed curves of Int $g_{k} \cap \hat{T}$ that bound disks in $\hat{T}$.

Assume, for either $k=i$ or $j$, Int $g_{k} \cap \hat{T}$ contains a simple closed curve that is essential on $\hat{T}$. Then an innermost such curve on $g_{k}$ bounds a meridional disk $D$ in the amber solid torus; amber since $g_{i}$ and $g_{j}$ each intersect every blue meridional disk. Because $\partial g_{i}$ and $\partial g_{j}$ are both parallel to $\partial D$, they bound disjoint amber meridional disks $D_{i}$ and $D_{j}$. These disks may be taken to have interiors disjoint from $g_{i}$ and $g_{j}$. Then $g_{i} \cup D_{i}$ and $g_{j} \cup D_{j}$ are disjoint embedded projective planes in a lens space. This cannot occur.

Since $K$ intersects each $g_{i}$ and $g_{j}$ in a transverse blue arc, Int $g_{k} \cap \hat{T}$ cannot contain a simple closed curve that is essential on $g_{k}$. Therefore Int $g_{k} \cap \hat{T}=\emptyset$. Then $\partial g_{i}$ and $\partial g_{j}$ cobound an annulus $A$ on $\hat{T}$ so that together $g_{i} \cup A \cup g_{j}$ forms a Klein bottle embedded in the blue solid torus. This cannot occur.

Lemma 5.4. Let $f$ be the face of a Scharlemann cycle of length 3 with label pair $\{i, i+1\}$ contained in the Heegaard solid torus $V$ whose edges do not lie in a disk on $\hat{T}$. It induces the subgraph $\Gamma$ of $G_{T}$ on $\hat{T}$ as indicated in figure 6 up to homeomorphism. Assume $H_{i, i+1}$ is unknotted. Then with respect to $\Gamma$ the meridian $m$ of a meridional disk of $V$ that is disjoint from $H_{i, i+1}$ appears on $\hat{T}$ as shown in figure 7(a) (up to Dehn twists along an essential simple closed curve $C$ of $\hat{T}$ that is disjoint from $\Gamma$ ).

Proof. Figure 6 shows $f$ with a labeling of its corners and the induced labeling of the endpoints of the edges of $\Gamma$. Let $C$ be an essential simple closed 


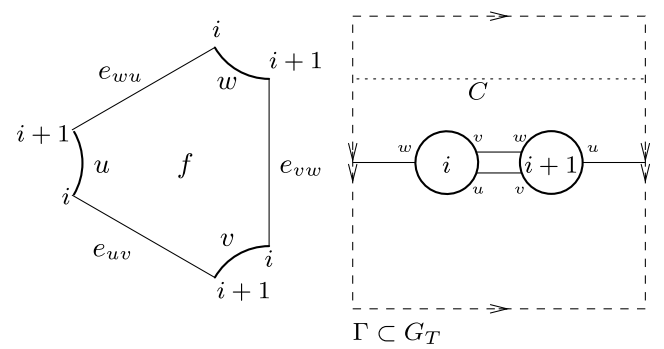

Figure 6: The face of a Scharlemann cycle of length 3 with labeled corners and its edges on $\hat{T}$.

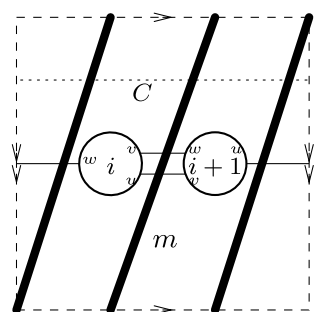

(a)

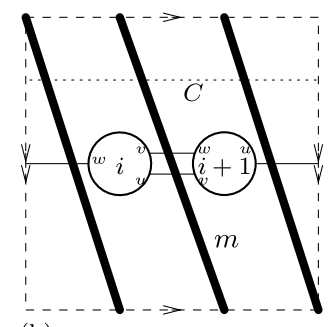

(b)

Figure 7: A priori, two possible meridians for $V$ up to Dehn twists along $C$. The viable one is shown in figure (a).

curve on $\hat{T}$ that is disjoint from $\Gamma$. Lemma 5.1 implies the existence of such a curve. Since $f \subset V$, Int $f \cap \hat{T}=\emptyset$. Thus by Lemma $5.2 \Delta(m, C)=3$. Therefore, up to Dehn twists along $C$, there are two possibilities for $m$. These two possibilities are illustrated in figure 7 .

Let $D$ be a meridional disk of $V$ bounded by $m$. By isotopies of $(D, \partial D)$ in $(V, \partial V)$ (thus keeping $m$ in $\hat{T})$, minimize $\left(\left|D \cap H_{i, i+1}\right|,|D \cap f|\right)$ lexicographically. Since $H_{i, i+1}$ is unknotted, it is straightforward to check that once minimized $\left|D \cap H_{i, i+1}\right|=0$ and $|D \cap f|=2$. The two intersections of $D \cap f$ are arcs on $f$ connecting $e_{w u}$ to the other two edges, see figure 8 . Hence, figure 7 also shows the two possibilities of how $m$ intersects $\Gamma$. On $D$ these two arcs cut off disjoint subdisks. Let $d$ be the one cut off by the arc on $f$ connecting $e_{w u}$ to $e_{v w}$. Then $d \cap m$ is an arc on $\hat{T}$ that joins $e_{v w}$ to $e_{w u}$ at the point nearer vertex $i+1$ than the other intersection of $m$ with $e_{w u}$. Therefore, $m$ must lie as in figure 7 (a) (up to Dehn twists along $C$ ).

Lemma 5.5. Let $\sigma$ be a Scharlemann cycle of length $p$ in $G_{S}$, and let $f$ be the face of $G_{S}$ bounded by $\sigma$. If the edges of $\sigma$ lie in a disk then $r=p$. 

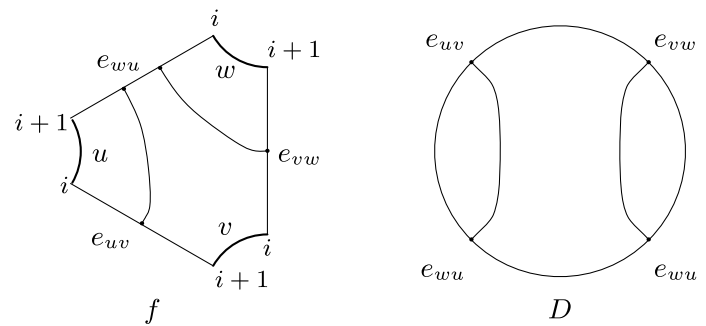

Figure 8: The two arcs of intersection of the meridional disk $D$ with the face $f$.

Furthermore, Int $f \cap \hat{T} \neq \emptyset$, and there must be a component of Int $f \cap \hat{T}$ that is essential on $\hat{T}$.

Proof. Let $\delta$ be a small disk in which the edges of $\sigma$ lie. Let $\{i, i+1\}$ be the label pair of $\sigma$.

Since lens spaces (other than $S^{1} \times S^{2}$ ) are irreducible, disk exchanges may be performed to replace $\hat{T}$ with another Heegaard torus $\hat{T}^{\prime}$ such that Int $f \cap \hat{T}^{\prime}$ contains only curves which are essential on $\hat{T}^{\prime}$. Similarly $\delta$ may be replaced with a disk $\delta^{\prime}$ in $\hat{T}^{\prime}$ such that the edges of $\sigma$ lie in $\delta^{\prime}$ and Int $f \cap \delta^{\prime}=\emptyset$. Then $N\left(\delta^{\prime} \cup H_{i, i+1} \cup f\right)$ forms a punctured lens space of order $p$. Hence $r=p$.

If all components of Int $f \cap \hat{T}$ were trivial on $\hat{T}$ then Int $f \cap \hat{T}^{\prime}=\emptyset$. Then $N\left(\delta^{\prime} \cup H_{i, i+1} \cup f\right)$ would form a punctured lens space within a neighborhood of one of the Heegaard solid tori. This cannot occur.

Lemma 5.6 (cf. Lemma 2.3, [31]). Let $\sigma$ be a Scharlemann cycle of length $p$ in $G_{S}$ with label pair $\{i, i+1\}$ and let $f$ be the face of $G_{S}$ bounded by $\sigma$. Suppose that $p \neq r$. Then the edges of $\sigma$ cannot lie in a disk in $\hat{T}$. Furthermore if Int $f \cap \hat{T} \neq \emptyset$ then the components of Int $f \cap \hat{T}$ are trivial curves in $\hat{T}$.

Proof. The proof of Lemma 2.3 of [31] works for our purposes. We have phrased our lemma to skirt Lemma 2.2 of [31]. Let us sketch the argument:

Assume $\sigma$ lies in a disk $D$ on $\hat{T}$. By Lemma $5.5 p=r$, contrary to our hypothesis that $p \neq r$. Therefore $\sigma$ cannot lie in a disk.

If Int $f \cap \hat{T}$ contains a curve which is essential on $\hat{T}$ then $\sigma$ must lie in an essential annulus $A$. Then, after suitable disk exchanges with $\hat{T}$, we may assume that there is a curve of Int $f \cap \hat{T}$ that bounds a disk $\delta$ in $f$ which is a meridional disk of one of the solid tori bounded by $\hat{T}$. We may then find 
an embedded annulus $A^{\prime}$ which connects $\partial \delta$ and a component of $\partial A$ that is otherwise disjoint from $A \cup \delta \cup H_{i, i+1} \cup f$. Push $\delta$ slightly off $f$ towards $A^{\prime}$. Then $N\left(A^{\prime} \cup A \cup \delta \cup H_{i, i+1} \cup f\right)$ is a punctured lens space of order $p$. This cannot occur within a lens space of order $r \neq p$.

Lemma 5.7. Let $f_{2}$ and $f_{3}$ be disk faces of $G_{S}$ bounded by Scharlemann cycles $\sigma_{2}$ and $\sigma_{3}$ of length 2 and 3 , respectively, such that $f_{2}$ and $f_{3}$ have the same color and $\sigma_{2}$ and $\sigma_{3}$ have the same label pair $\{i, i+1\}$. Let $V$ be the solid torus bounded by $\hat{T}$ of the same color as $f_{2}$ and $f_{3}$. Let $m$ be its meridian. Then

(1) $\sigma_{2}$ and $\sigma_{3}$ each lie in an essential annulus $A_{2}$ and $A_{3}$, respectively, on $\hat{T}$

(2) two edges of $\sigma_{3}$ and one edge of $\sigma_{2}$ are mutually parallel in the subgraph of $G_{T}$ consisting of the edges $\sigma_{2} \cup \sigma_{3}$ and the vertices $i$ and $i+1$,

(3) the cores $C_{2}$ and $C_{3}$ of the annuli $A_{2}$ and $A_{3}$, respectively, have distance 1 on $\hat{T}$ and

(4) the curves $m, C_{2}$ and $C_{3}$ may be oriented so that $[m]=3\left[C_{2}\right]+2\left[C_{3}\right] \in$ $H_{1}(\hat{T})$.

Proof. By Lemma 5.6 for at least one of $p=2$ or $p=3$ the edges $\sigma_{p}$ cannot lie in a disk on $\hat{T}$. Then by Lemma 5.1 the edges of $\sigma_{p}$ must lie in an essential annulus $A_{p}$ on $\hat{T}$. Let $p^{\prime} \in\{2,3\}-\{p\}$. The proof of this lemma is now broken into the following three claims.

Claim 5.1. The edges of $\sigma_{p^{\prime}}$ lie in an essential annulus $A_{p^{\prime}}$ on $\hat{T}$.

Proof. Assume the edges of $\sigma_{p^{\prime}}$ lie in an disk. Then by Lemma 5.5 both $r=p^{\prime}$ and there must be a curve of Int $f_{p^{\prime}} \cap \hat{T}$ that is essential in $\hat{T}$. Because $r \neq p$, Lemma 5.6 implies that Int $f_{p} \cap \hat{T}$ may only contain curves that are trivial in $\hat{T}$. Perform disk exchanges to pass to a new Heegaard torus that is disjoint from Int $f_{p}$ and intersects Int $f_{p^{\prime}}$ in only simple closed curves which are essential on the Heegaard torus. We will retain the former names for all our objects that may have been affected by these disk exchanges.

By Lemma 5.1 now $M=N\left(A_{p} \cup H_{i, i+1} \cup f_{p}\right)$ is a solid torus such that the core of $A_{p}$ runs $p$ times in the longitudinal direction of $M$. Also, now an innermost curve $\xi$ of Int $f_{p^{\prime}} \cap \hat{T}$ will bound a disk on $f_{p^{\prime}}$ that is a meridional disk of one of the solid tori bounded by $\hat{T}$. Since $\xi$ is essential on $\hat{T}$ it must be parallel to $A_{p}$. Let $A^{\prime}$ be an annulus on $\hat{T}$ giving a parallelism between $\xi$ and $A_{p}$. Note that $A^{\prime}$ is disjoint from $f_{p}$. Then $N\left(\delta \cup A^{\prime} \cup A_{p} \cup H_{x, x+1} \cup f_{p}\right)$ 


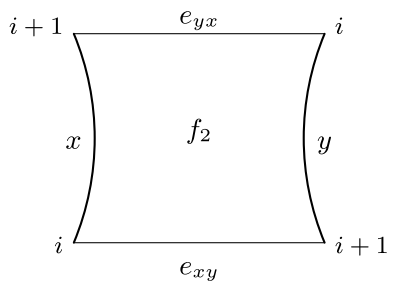

Figure 9: The face $f_{2}$ of a Scharlemann cycle of length 2 .

may be viewed as a two-handle $N\left(\delta \cup A^{\prime}\right)$ attached to $M$ along the core of $A_{p}$. This produces a punctured lens space of order $p$ in our lens space of order $p^{\prime}$. This is a contradiction.

We may identify $f_{3}$ and the subgraph of $G_{T}$ induced by $\sigma_{3}$ with $f$ and $\Gamma$ of figure 6 so that $\sigma_{3}=\left\{e_{u v}, e_{v w}, e_{w u}\right\}$. Label the edges and corners of $f_{2}$ as shown in figure 9 so that $\sigma_{2}=\left\{e_{x y}, e_{y x}\right\}$. By Claim 5.1 above, the edges $\sigma_{3}$ lie in the essential annulus $A_{3}$ on $\hat{T}$ and the edges $\sigma_{2}$ lie in the essential annulus $A_{2}$ on $\hat{T}$. Let $C_{2}$ and $C_{3}$ be cores of $A_{2}$ and $A_{3}$, respectively.

Claim 5.2. Ignoring the curve $m$, the subgraph of $G_{T}$ induced by the edges $\sigma_{2} \cup \sigma_{3}$ appears as in figure 10. Furthermore, $\Delta\left(C_{2}, C_{3}\right)=1$ on $\hat{T}$.

Proof. Since $\sigma_{2}$ does not lie in a disk, we may assume $e_{x y}$, say, does not lie between $e_{u v}$ and $e_{v w}$ in $G_{T}$. Therefore, the endpoint of $e_{x y}$ on vertex $i$ either (1) appears between $e_{u v}$ and $e_{w u}$ (i.e. it is separated from the endpoint of $e_{v w}$ on vertex $i$ by the endpoints of $e_{u v}$ and $e_{w u}$ ) or (2) between $e_{v w}$ and $e_{w u}$. Since the corner $x$ of $f_{2}$ on $\partial_{v} H_{i, i+1}$ is disjoint from the corners $u, v$, and $w$ of $f_{3}$, we may follow it along $\partial_{v} H_{i, i+1}$ from the endpoint of $e_{x y}$ on

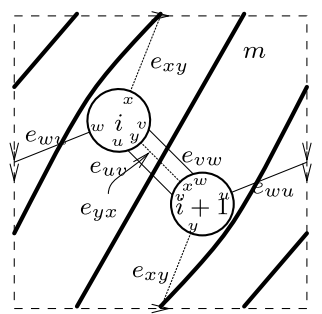

(i)

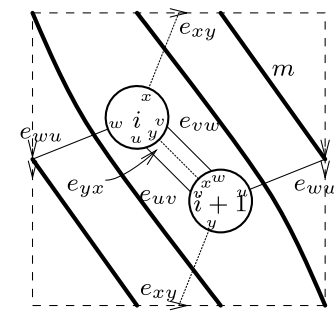

(ii)

Figure 10: Two possibilities for the meridian $m$ of a solid torus that contains the faces of Scharlemann cycles $\sigma_{3}=\left\{e_{u v}, e_{v w}, e_{w u}\right\}$ and $\sigma_{2}=\left\{e_{x y}, e_{y x}\right\}$ on $G_{T}$. 
vertex $i$ to the endpoint of $e_{y x}$ on vertex $i+1$ by following the corners of $f_{3}$ that flank it.

In case (1), the corner $x$ lies between the corners $w$ and $u$ on $\partial_{v} H_{i, i+1}$ and so the endpoint of $e_{y x}$ on vertex $i+1$ must lie between $e_{v w}$ and $e_{w u}$. Then in order for $e_{x y}$ and $e_{y x}$ to both have endpoints on the corner $y$ of $f_{2}$, this corner must also lie between the corners $w$ and $u$. Consequentially, the cores $C_{2}$ and $C_{3}$ are isotopic on $\hat{T}$. If (Int $\left.f_{2} \cup \operatorname{Int} f_{3}\right) \cap \hat{T} \neq \emptyset$ then by Lemma 5.6 the intersection may only consist of simple closed curves that are trivial on $\hat{T}$. Eliminate these intersections by disk exchanges of $\hat{T}$ so that $\left(\operatorname{Int} f_{2} \cup \operatorname{Int} f_{3}\right) \cap \hat{T}=\emptyset$. Then by Lemma 5.2 for the meridian $m$ of the solid torus containing $f_{2}$ and $f_{3}, \Delta\left(m, C_{2}\right)=2$ and $\Delta\left(m, C_{3}\right)=3$. This cannot occur since $C_{2}$ and $C_{3}$ are isotopic.

In case (2), the corner $x$ lies between the corners $w$ and $v$ on $\partial_{v} H_{i, i+1}$ and so the endpoint of $e_{y x}$ on vertex $i+1$ must lie between $e_{u v}$ and $e_{v w}$. Therefore, the edge $e_{y x}$ lies in the bigon of $T$ bounded by $e_{u v}$ and $e_{v w}$. The corner $y$ then lies between the corners $u$ and $v$ on $\partial_{v} H_{i, i+1}$ so that the endpoint of $e_{x y}$ on vertex $i+1$ lies between $e_{u v}$ and $e_{w u}$. Observe that $\Delta\left(C_{2}, C_{3}\right)=1$. This completes the claim.

Claim 5.3. The curves $m, C_{2}$, and $C_{3}$ may be oriented so that $[m]=$ $3\left[C_{2}\right]+2\left[C_{3}\right] \in H_{1}(\hat{T})$.

Proof. Because $\Delta\left(C_{2}, C_{3}\right)=1$ by Claim 5.2, (Int $\left.f_{2} \cup \operatorname{Int} f_{3}\right) \cap \hat{T}$ may only consist of curves that are trivial on $\hat{T}$. As in the proof of Claim 5.1, perform disk exchanges to pass to a new Heegaard torus that is disjoint from Int $f_{2} \cup$ Int $f_{3}$ and retain the former nomenclature. Then Lemma 5.2 implies that $\Delta\left(C_{2}, m\right)=2$ and $\Delta\left(C_{3}, m\right)=3$. Hence, with respect to $C_{2}$ and $C_{3}$, there are only two possibilities for the (unoriented) isotopy class of $m$ as described in the claim.

These three claims complete the lemma. The possible arrangements of the edges $\sigma_{2}=\left\{e_{x y}, e_{y x}\right\}$ and $\sigma_{3}=\left\{e_{u v}, e_{v w}, e_{w u}\right\}$ with the meridian $m$ of $V$ are shown in figure 10 .

Lemma 5.8. Continuing Lemma 5.7, if we disregard the other arcs of $K \cap$ $V$, the arc $K_{i, i+1}$ is isotopic rel- $\partial$ in $V$ to an arc in $\hat{T}$ with interior disjoint from the edges $\sigma_{2} \cup \sigma_{3}$ of $G_{T}$ that is incident to each vertex of $G_{T}$ between the consecutive edges of $\sigma_{3}$. See figure 11 .

Proof. Among meridional disks of $V$ that are disjoint from $K_{i, i+1}$ assume $D$ is one that intersects $f_{2} \cup f_{3}$ minimally. Consider an arc $\gamma$ of $D \cap\left(f_{2} \cup f_{3}\right)$ 


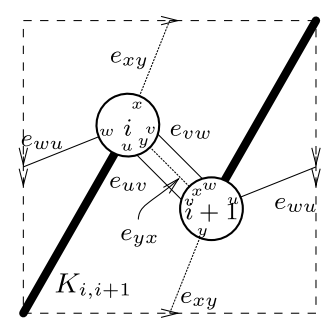

Figure 11: The isotopy rel- $\partial$ of $K_{i, i+1}$ onto $\hat{T}$.

that is outermost on $D$ and the disk $\delta$ that it sections off. By minimality, $\gamma$ cannot be boundary parallel on $f_{2} \cup f_{3}$ to an edge of these faces. Because the two edges $e_{u v}$ and $e_{v w}$ of $\sigma_{3}$ flank the edge $e_{y x}$ of $\sigma_{2}$ in $G_{T}, \gamma \subset D \cap f_{3}$; $\gamma$ could not be outermost on $D$ otherwise. Since $\gamma$ is not parallel to an edge of $f_{3}$ it must be parallel to a corner. Join this rectangle of parallelism on $f_{3}$ to $\delta$ and perform a slight isotopy to create a bigon $B$ that has one edge on $\partial_{v} H_{i, i+1}$, has its other edge on $\hat{T}$, and is otherwise disjoint from $H_{i, i+1} \cup f_{2} \cup f_{3}$. Because $B$ is disjoint from $f_{2} \cup f_{3}$ and two edges of $\sigma_{3}$ flank an edge of $\sigma_{2}$ in $G_{T}$, the edge of $B$ on $\partial_{v} H_{i, i+1}$ must lie between the two consecutive corners of $f_{3}$. Thus, $K_{i, i+1}$ is isotopic rel- $\partial$ to the edge of $B$ on $\hat{T}$ as shown in figure 11 .

Lemma 5.9. Let $f$ and $f^{\prime}$ be the faces of two order 3 Scharlemann cycles in $G_{S}$ of the same color and with the same label pair $\{i, i+1\}$. Assume Int $\left(f \cup f^{\prime}\right) \cap \hat{T}=\emptyset$. Let $V$ be the solid torus that contains $f$ and $f^{\prime}$. Then $f$ and $f^{\prime}$ are parallel in $V$ - Int $H_{i, i+1}$.

Proof. Since $M=V-\left(N(f) \cup \operatorname{Int} H_{i, i+1}\right)$ is a solid torus on which $\hat{T} \cap \partial M$ is the disjoint union of a disk $D$ and an annulus $A$ that is longitudinal. Furthermore $\partial_{v} H_{i, i+1} \cap \partial M$ is three rectangles, one connecting the two components of $\partial A$ and the other two connecting the two components of $\partial A$ to $\partial D$. Since $f^{\prime}$ is a properly embedded disk in $M$ (with boundary in $\left.\left(\hat{T} \cup \partial_{v} H_{i, i+1}\right) \cap \partial M\right)$, it must either be boundary parallel or a meridional disk of $M$.

Since $\partial f^{\prime}$ traverses the rectangles of $\partial_{v} H_{i, i+1} \cap \partial M$ a total of three times it either traverses the three rectangles each once or it traverses the rectangle connecting the two components of $\partial A$ thrice. But since one of these rectangles may be traversed by $\partial f^{\prime}$ only in a single direction, if the latter occurs then $\partial f^{\prime}$ intersects $A$ in three spanning arcs; no disk in a solid torus has such a boundary. Therefore, the former case occurs and $\partial f^{\prime}$ must intersect 
$A$ twice (and $D$ once). Therefore $f^{\prime}$ cannot be a meridional disk. Hence $f^{\prime}$ is boundary parallel, and it thus follows that $f^{\prime}$ is parallel to $f$.

Lemma 5.10. Assume $t=2$. If $s>2$ then the interior of a bigon of $G_{S}$ is disjoint from $\hat{T}$. If $s>3$ then the interior of a trigon of $G_{S}$ is disjoint from $\hat{T}$.

Proof. Let $\sigma$ be two or three edges of $G_{S}$ that bound a $p$-gon face $f$ with Int $f \cap \hat{T} \neq \emptyset, p=2$ or 3 , respectively. Because $t=2, \sigma$ is a Scharlemann cycle of length $p$. Since $r \geq s>p$, by Lemma 5.6 the edges of $\sigma$ do not lie in a disk. By Lemma 5.1 the edges of $\sigma$ lie in an essential annulus $A$ on $\hat{T}$. Furthermore by Lemma 5.6 the components of $\operatorname{Int} f \cap \hat{T}$ are trivial curves in $\hat{T}$. Indeed, since $t=2$ these components must be trivial curves in $T$; otherwise $G_{T}$ would lie in a disk. Since lens spaces are prime and $K$ is not contained in a three-ball, we may isotop Int $f$ to remove its intersections with $\hat{T}$. This reduces $|S \cap T|$ contrary to our minimality assumptions.

\section{6. $s \geq 3$ and $t=2$}

This section is devoted to proving the following theorem.

Theorem 6.1. If $s \geq 3$ then $s=3$ and $K$ is a knot in $W(-3,-3+1 / k) \cong$ $L(9 k-3,3 k-2)$ arising as the core of the -3 surgery for some integer $k \neq 0$ (and for each integer $k \neq 0$ there is such a knot). Equivalently, up to homeomorphism, $K$ is the $|3 k-1|$ th grid number 1 knot in $L(9 k-3,3 k-$ $2)$. Furthermore $E(K)$ is fibered with fiber $S$ if and only if $k= \pm 1$ :

- If $k=+1$ then $K$ is a torus knot in $L(6,1)$ with monodomy $\phi \cong \tau_{x}^{2} \tau_{y}$.

- If $k=-1$ then $K$ is a non-torus knot in $L(12,5)$ monodromy $\phi \cong \tau_{x}^{4} \tau_{y}$.

Proof. We give an overview of the proof. Assume $s \geq 3$. Theorem 1.1 of [33] states that a knot in a lens space with an essential once-punctured genus $g$ surface properly embedded in its exterior such that the boundary of the surface is distance at least $4 g-1$ from the meridian (i.e. $4 g-1 \leq s$ ) is onebridge with respect to the genus one Heegaard splitting of the lens space. Since our surface $S$ has genus 1 and we are assuming $s \geq 3$, it follows that $K$ is one-bridge. Therefore, $t=2$.

With $t=2$, the first three subsections here conclude that $s=3$ and that $G_{S}$ must appear as in figure 18(b). Then Lemma 6.10 describes the lens spaces which contain knots with such surfaces $S$. Lemma 6.11 identifies 


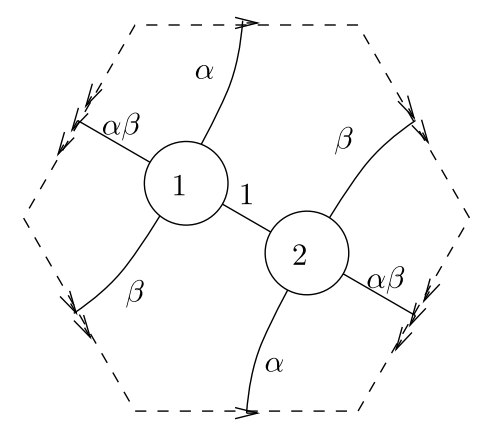

Figure 12: The four edge class labels of $G_{T}$.

each knot $K$ as grid number 1 . Lemma 6.12 gives the surgery description of this family of knots. Lemma 6.13 shows that $E(K)$ is fibered if and only if $k= \pm 1$ and that $S$ is an incompressible if and only if $k \neq 0$. Lemma 6.14 shows that if $k=+1$ then $K$ is a torus knot. Lemma 6.15 shows that if $k=-1$ then $K$ has fibered exterior with monodromy $\phi \cong \tau_{x}^{4} \tau_{y}$.

Throughout this section we assume $s \geq 3$. As stated in the proof above for use in the ensuing lemmas, $K$ is therefore one-bridge. Hence $K=K_{a} \cup$ $K_{b}$ where $K_{a}$ and $K_{b}$ are unknotted arcs in $V_{a}$ and $V_{b}$, respectively. Consequentially, $t=2$.

Since $t=2$, the graph $G_{T}$ has two vertices and $s$ edges. By the Parity rule, there are at most four edge classes in $G_{T}$ which we denote $1, \alpha, \beta, \alpha \beta$ (see Section 4 of [31] and [34]) as shown in figure 12. Label an edge of $G_{S}$ by the class of the corresponding edge in $G_{T}$. The label of an edge is called its edge class label.

\subsection{Bigons of $G_{S}$}

The following three lemmas give strong restrictions on the graph $G_{S}$.

Lemma 6.1 (Lemma 4.1, [31]). Since $s>2$, any two bigons in $G_{S}$ of the same color have the same pair of edge class labels. Furthermore, the two bigons are parallel.

Proof. Since the interior of a bigon is disjoint from $\hat{T}$ by Lemma 5.10, the proof of Lemma 5.2 [34] continues to hold for us too. Let us sketch the argument.

Let $B_{1}$ and $B_{2}$ be two bigons of $G_{S}$ in, say, $V_{a}$. Recall $H_{1,2}$ is the onehandle neighborhood of $K$ in $V_{a}$. Then $M=V_{a}-\left(N\left(B_{1}\right) \cup \operatorname{Int} H_{1,2}\right)$ is a 
solid torus on which $\hat{T} \cap \partial M$ is a longitudinal annulus. Since $B_{2}$ is a properly embedded disk in $M$, it must either be boundary parallel or a meridional disk. A meridional disk will meet $\partial M-\hat{T}$ minimally once. Because the corners of $B_{2}$ must cross $\partial_{v} H_{1,2}$ twice, $B_{2}$ must be boundary parallel. It follows that $B_{2}$ is indeed parallel to $B_{1}$. Hence it has the same pair of edge class labels.

Lemma 6.2 (cf. Lemma 4.2, [31]). Parallel edges of $G_{S}$ have distinct edge class labels.

Proof. Assume two parallel edges $e_{1}$ and $e_{2}$ of $G_{S}$ have the same edge class labels with no third edge in between also having the same edge class label. By Lemma 5.6 these two edges cannot be adjacent in $G_{S}$. Because Lemma 6.1 implies that two bigons of the same color must have the same pair of edge class labels, there may be at most two edges between $e_{1}$ and $e_{2}$ in $G_{S}$. If there are these two edges between $e_{1}$ and $e_{2}$, then they must have the same edge class label. This however contradicts Lemma 5.6. Therefore there is necessarily just one edge $e_{1 \frac{1}{2}}$ of $G_{S}$ between $e_{1}$ and $e_{2}$, and this edge has a different edge class label than that of $e_{1}$ and $e_{2}$.

Let $B_{i}$ be the bigon of $G_{S}$ bounded by $e_{i}$ and $e_{1 \frac{1}{2}}$. Extend $B_{1}$ and $B_{2}$ radially to the core $K$ of $H_{1,2} \cup H_{2,1}$ to form two Möbius bands $A_{1}$ and $A_{2}$ respectively on either side of the torus $\hat{T}$. Since $e_{1}$ and $e_{2}$ are in the same edge class, $\partial A_{1} \cup \partial A_{2}$ bounds a bigon $B \subset \hat{T}$. Thus $A_{1} \cup A_{2} \cup B$ is a Klein bottle $R$ in our lens space $X$ on which $K$ lies. Moreover, $K$ lies as an essential curve on $R$ and $R-N(K)$ is an annulus. Since the curves of $\partial(R-N(K))$ have induced coherent orientations on $\partial N(K)$, then $2[K]=0$ in $H_{1}(X)$. Hence $s=2$ contrary to our assumption that $s \geq 3$.

Remark 6.1. Lemma 4.2 of [31] appeals to a hypothesis that $K$ is hyperbolic for its proof. Our proof uses that $s \geq 3$ instead.

Lemma 6.3 (Lemma 4.3, [31]). Since $s>2, G_{S}$ cannot contain more than three mutually parallel edges.

Proof. This is an easy consequence of Lemmas 6.1 and 6.2.

\subsection{If $G_{S}$ has 3 mutually parallel edges}

Since $S$ is a once-punctured torus, $G_{S}$ may have at most three edge classes. By Lemma 6.3 there are at most three edges in any $G_{S}$-edge class. In this 


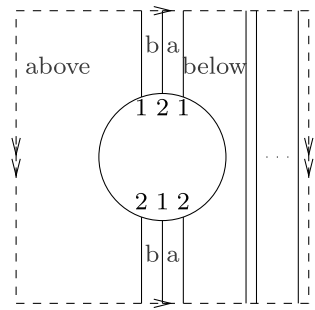

(a)

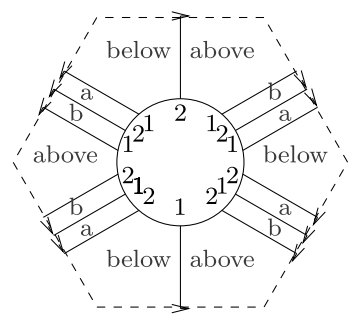

(c)

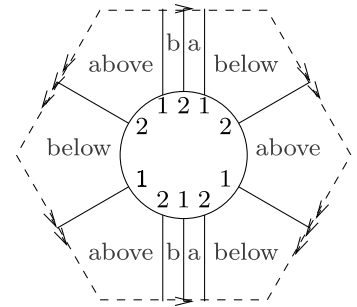

(b)

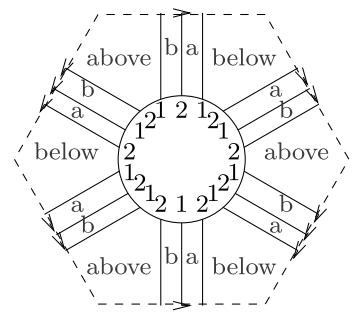

(d)

Figure 13: If $G_{S}$ has three mutually parallel edges, no set of more than three mutually parallel edges, and obeys the Parity rule then, a priori, $G_{S}$ may be one of the four shown.

subsection, we assume at least one $G_{S^{-}}$edge class does contain three mutually parallel edges.

Lemma 6.4. If $s \geq 3$ and $K$ is not a torus knot then $G_{S}$ cannot have three mutually parallel edges.

Proof. After enumerating all possibilities for $G_{S}$ having at most three edges in any $G_{S}$-edge class (and three edges in at least one $G_{S}$-edge class) and eliminating those that violate the Parity rule, there are four left. These possibilities are shown in figure 13.

We eliminate the remaining possibilities with Claims 6.1, 6.2, 6.3, and Lemma 6.5 below.

Claim 6.1. Figure 13(a) cannot occur.

Proof. In figure 13(a), there must be an odd (and hence nonzero) number of essential simple closed curves of $S \cap T$ on $G_{S}$ in order to achieve the checkerboard coloring. Such simple closed curves must also be essential in $T$ since $G_{T}$ does not lie in a disk and $S$ is incompressible. However, since Lemma 6.2 states that the three parallel edges of $G_{S}$ must each have different 
$G_{T}$-edge class labels, the complement of these three $\operatorname{arcs}$ on $T$ must be a disk. Hence, there can be no essential simple closed curve of $S \cap T$ on $T$. This is a contradiction. Figure 13(a) cannot occur.

The remaining three possibilities for $G_{S}$ each contain for each color a trigon and one, two or three bigons. By Lemma 6.2 the three $G_{T}$-edge class labels of three mutually parallel edges of $G_{S}$ must be distinct. Then by Lemma 6.1 the $G_{T}$-edge class labels for one set of three mutually parallel edges determines the $G_{T}$-edge class labels for every other set of three mutually parallel edges. Furthermore, by Lemma 5.7 a trigon of $G_{S}$ must have two edges in the same $G_{T}$-edge class as an edge of a bigon of $G_{S}$ of the same color. This then implies that the remaining two edges of the trigon and bigon are labeled with two other distinct $G_{T}$-edge class labels.

Claim 6.2. Figure 13(c) cannot occur.

Proof. After labeling the sets of three mutually parallel edges in accordance with Lemmas 6.2 and 6.1, there is no choice of label for the final edge that is consistent with Lemma 5.7.

Claim 6.3. Figure 13(d) cannot occur.

Proof. The pattern of $G_{T}$-edge class labels forced upon figure 13(d) by the sets of three mutually parallel edges causes the two trigons to have all three edges with the same $G_{T}$-edge class label. Because in this case $r \geq s=9$, this contradicts Lemma 5.6.

Lemma 6.5. If $G_{S}$ appears as in figure 13(b), then $K$ is a torus knot.

Proof. Assume $G_{S}$ does appear as in figure 13(b). There are two Scharlemann cycles of length $3, \sigma_{3 a}$ and $\sigma_{3 b}$, bounding amber and blue faces $f_{3 a}$ and $f_{3 b}$, respectively; there are two Scharlemann cycles of length $2, \sigma_{2 a}$ and $\sigma_{2 b}$, bounding amber and blue faces $f_{2 a}$ and $f_{2 b}$, respectively.

By Lemma 5.7 we may assume $\sigma_{3 a}=\left\{e_{u v}, e_{v w}, e_{w u}\right\}$ and $\sigma_{2 a}=\left\{e_{x y} e_{y x}\right\}$ labels the edges as in figure 10. As there are only these five edges in $S \cap T$, Lemma 5.7 also implies that $\sigma_{3 b}=\left\{e_{u v}, e_{v w}, e_{x y}\right\}$ and $\sigma_{2 b}=\left\{e_{w u}, e_{y x}\right\}$. With this labeling, $G_{T}$ and $G_{S}$ appear as in figure 14.

Lemma 5.7 describes how the meridians of $V_{a}$ and $V_{b}$ may appear on $\hat{T}$ with respect to $G_{T}$. The amber meridian $m_{a}$ appears as $m$ in figure 10(i) or (ii). Similarly, the blue meridian $m_{b}$ appears on $\hat{T}$ with respect to $G_{T}$ as a reflection of $m$ across a line of slope 1 in figure 10(i) or (ii). 

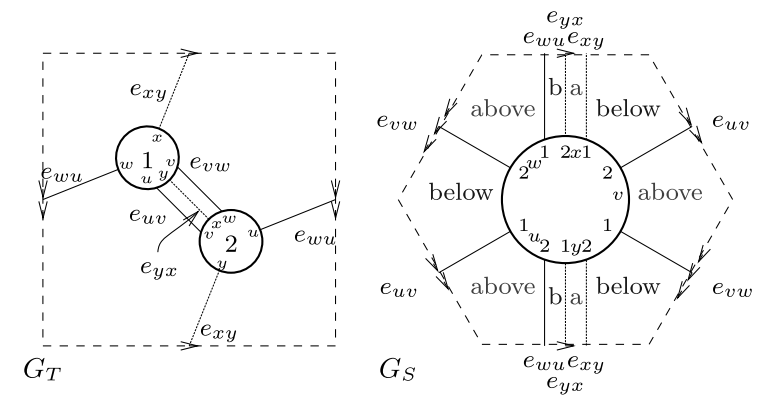

Figure 14: Labels for figure 13(b).

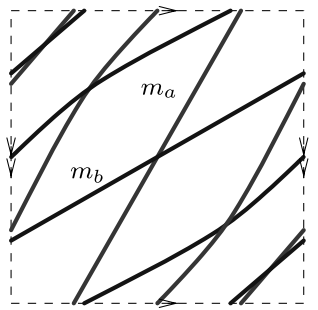

(i)

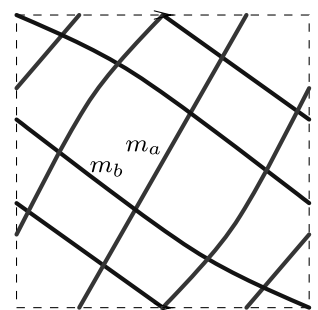

(iii)

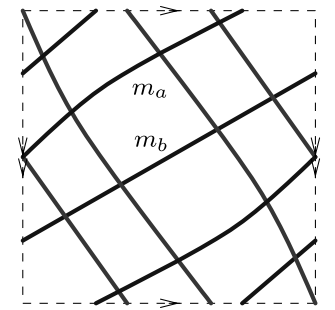

(ii)

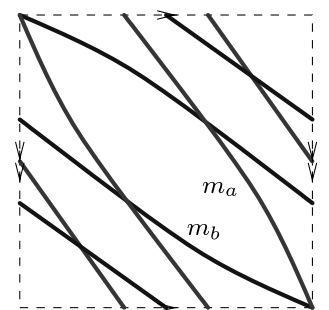

(iv)

Figure 15: The four possibilities considered in Lemma 6.5 for the two meridians of the Heegaard solid tori for figure 14.

Put together, the four possibilities for $m_{a}$ and $m_{b}$ on $\hat{T}$ are shown in figure 15. The latter two are reflections of the former two. In (ii) and (iii), $m_{a}$ and $m_{b}$ define a lens space of order $r=13$ which cannot contain our knot with $s=5$. The meridians $m_{a}$ and $m_{b}$ in (i) and (iv) each define the lens space $L(5,1)$ which may contain our knot. These meridians (in relation to $G_{T}$ ) are both of the type shown in figure 10(i).

Lemma 5.8 allows us to obtain a diagram of $K$ on $\hat{T}$ in relationship to $G_{T}$. The amber arc $K_{a}$ of $K$ is isotopic rel- $\partial$ in $V_{a}$ to an arc in $\hat{T}$ with interior disjoint from the edges of $G_{T}$ that is incident to each vertex of $G_{T}$ between the consecutive edges of $\sigma_{3 a}$. Similarly, the blue arc $K_{b}$ of $K$ is 


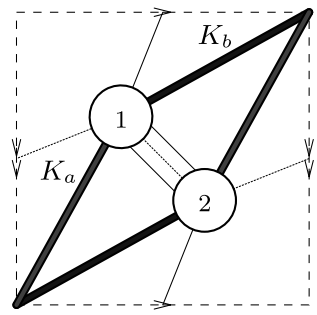

(i)

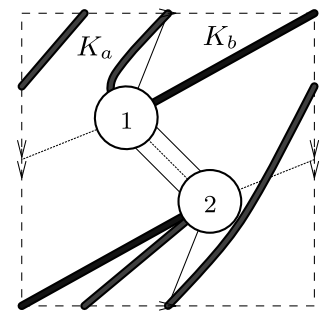

(ii)

Figure 16: (i) Isotopies of $K_{a}$ and $K_{b}$ onto $\hat{T}$. (ii) Result of isotopy of $K_{a}$ across a meridional disk of $V_{a}$ back onto $\hat{T}$. $K$ is thus a torus knot.

isotopic rel- $\partial$ in $V_{b}$ to an arc in $\hat{T}$ with interior disjoint from the edges of $G_{T}$ that is incident to each vertex of $G_{T}$ between the consecutive edges of $\sigma_{3 b}$. The result of these isotopies is shown in figure 16(i). Note that $K_{a}$ lies above $K_{b}$. The $\operatorname{arc} K_{a}$, say, now may be isotoped rel- $\partial$ across the meridional disk of $V_{a}$ so that it lies on $\hat{T}$ overlapping $K_{b}$ only at their endpoints as shown in figure 16(ii). Hence, $K$ is a torus knot.

Remark 6.2. In fact if $G_{S}$ does appear as in figure 13(b), then $K$ is a type $\{2,3\}$ torus knot in $L(5,1)$.

\subsection{If $G_{S}$ only has fewer than 3 mutually parallel edges}

We now may assume no edge class of $G_{S}$ has more than two mutually parallel edges.

Lemma 6.6. If $s \geq 3$ and $G_{S}$ does not have three mutually parallel edges then $G_{S}$ appears as in figure 18(b).

Proof. After enumerating all possibilities for $G_{S}$ and eliminating those that violate the Parity rule, there are five left. The two shown in figure 17 correspond to $s=1$ and $s=2$, respectively, contrary to our assumption that $s \geq 3$. Therefore, we are left to consider the three remaining possibilities shown in figure 18. We eliminate figure 18(c) in Lemma 6.7 and figure 18(a) in Lemma 6.8 below leaving figure $18(\mathrm{~b})$ as the only viable possibility.

We determine the knots $K$ with $G_{S}$ as in figure 18(b) in Section 6.4.

Lemma 6.7. $G_{S}$ cannot appear as in figure 18(c). 


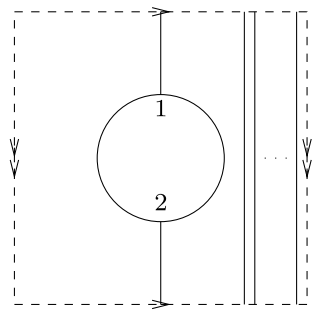

(a)

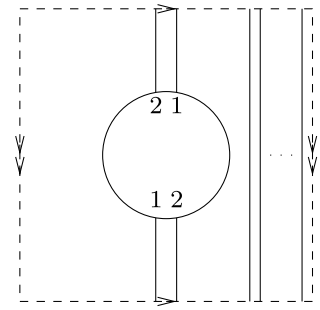

(b)

Figure 17: These two possibilities for $G_{S}$ have no set of three mutually parallel edges and obey the Parity rule, yet have $s<3$.

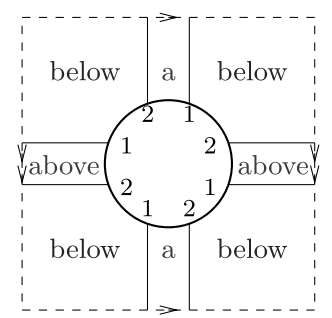

(a)

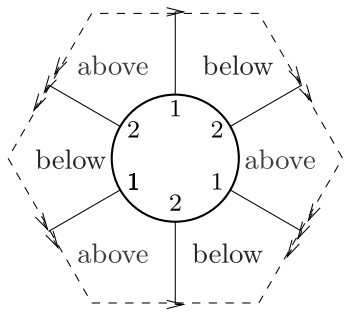

(b)

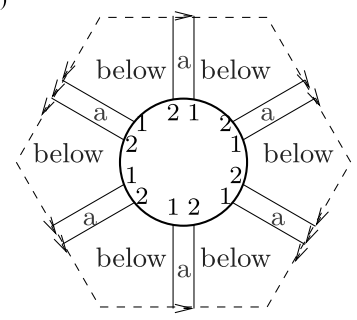

(c)

Figure 18: These are the three possibilities for $G_{S}$ that have no set of three mutually parallel edges, obey the Parity rule, and have $s \geq 3$.

Proof. Assume $G_{S}$ does appear as in figure $18(\mathrm{c})$. The graph $G_{S}$ contains two Scharlemann cycles of length 3 , say $\sigma_{3}$ and $\sigma_{3}^{\prime}$, of the same color and with the same label pair. Since $s=6$, by Lemma 5.10 the faces of $\sigma_{3}$ and $\sigma_{3}^{\prime}$ must have interior disjoint from $\hat{T}$. Then by Lemma 5.9 the faces of $\sigma_{3}$ and $\sigma_{3}^{\prime}$ are parallel. Hence the six edges of $G_{T}$ lie in an essential annulus on $\hat{T}$; four in one $G_{T}$-edge class and two in another.

The graph $G_{S}$ also contains three Scharlemann cycles of length 2, say $\sigma_{2}, \sigma_{2}^{\prime}$, and $\sigma_{2}^{\prime \prime}$, of the other color. By Lemma 6.1 all three have the same pair of $G_{T}$-edge class labels. Hence, three edges of $G_{T}$ lie in one $G_{T}$-edge class and the other three lie in another. This is a contradiction. 
Lemma 6.8. $G_{S}$ cannot appear as in figure 18(a).

Proof. Assume $G_{S}$ does appear as in figure 18(a). Then $G_{S}$ contains two Scharlemann cycles of length 2 , say $\sigma_{2}$ and $\sigma_{2}^{\prime}$, with faces $f_{2}$ and $f_{2}^{\prime}$, respectively, say colored amber and the same label pair. By Lemma 6.1 the faces of $\sigma_{2}$ and $\sigma_{2}^{\prime}$ must be parallel. Let $\sigma_{2}=\left\{e_{x y}, e_{y x}\right\}$ with corners $x$ and $y$ as in figure 9 (setting $i=1$ ). Similarly let $\sigma_{2}^{\prime}=\left\{e_{u v}, e_{v u}\right\}$ with corners $u$ and $v-$ replace $x$ with $u$ and $y$ with $v$ in figure 9 . Without loss of generality we may assume these edges and corners appear on $G_{S}$ and $G_{T}$ as shown in figure 19. The two pairs of parallel edges in $G_{T}$ hands us two bigons $D_{1}$ and $D_{2}$ on $T$.

The graph $G_{S}$ also contains a Scharlemann cycle $\sigma_{4}$ of length 4 bounding a blue face $f_{4}$. Its four edges coincide with those of the two order two Scharlemann cycles.

A simple closed curve $\gamma$ of Int $f_{4} \cap \hat{T} \neq \emptyset$ that is innermost on $f_{4}$ bounds a disk in $f_{4}$ and must also bound a disk in $T$. Otherwise $\gamma$ would be parallel on $\hat{T}$ to the core of the annulus in which the edges of $\sigma_{2}$ lie. By Lemma 5.1 $\gamma$ runs twice in the longitudinal direction of $V_{a}$, hence the disk in $f_{4}$ that $\gamma$ bounds lies in $V_{b}$. A neighborhood of this disk together with $V_{a}$ forms a punctured lens space of order 2 embedded in our lens space $X$. Hence, $s=2$ contrary to our assumptions. Since $\gamma$ bounds a disk in $T$ an isotopy of Int $S$ will reduce $|S \cap T|$. Hence, Int $f_{4} \cap \hat{T}=\emptyset$.

We may now form an embedded once-punctured Klein bottle $R^{\prime}$ in $V_{b}$ by attaching $D_{1}$ and $D_{2}$ to $f_{4}$ along their common edges. Since $\partial R^{\prime} \subset \partial_{v} H_{2,1}$ it must either be a meridian of $K$ or bound a disk on $\partial_{v} H_{2,1}$. In either case there is a properly embedded disk in $H_{2,1}$ with the same boundary as $R^{\prime}$. Together, $R^{\prime}$ and this disk form an embedded Klein bottle in the solid torus $V_{b}$. This cannot occur.
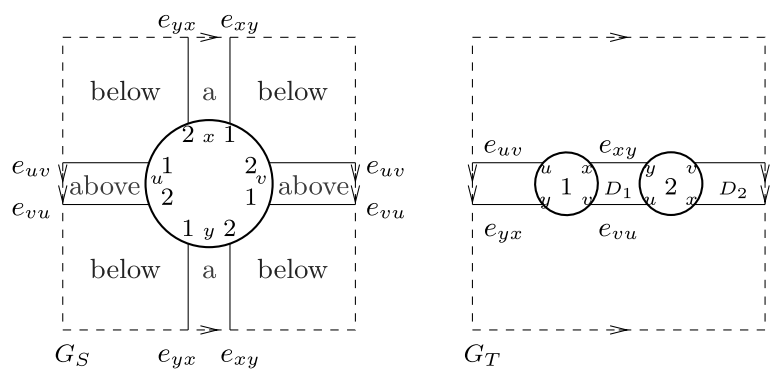

Figure 19: The edges of figure 18(a) on $G_{S}$ and $G_{T}$ with labelings. 


\subsection{When $G_{S}$ appears as figure $18(\mathrm{~b})$}

Lemma 6.9. If $G_{S}$ is as in figure $18(b)$ then $G_{T}$ does not lie in a disk on $\hat{T}$.

Proof. Assume $G_{T}$ lies in a disk $D$ in $\hat{T}$. By a sequence of disk exchanges, we may assume the interiors of the two faces of $G_{S}$ are disjoint from $D$. Then $M=N\left(D \cup H_{1,2} \cup H_{2,1}\right)$ is a genus 2 handlebody.

The two faces of $G_{S}$ are each faces of order 3 Scharlemann cycles. Attaching the two-handle neighborhoods of these two faces to $M$ will produce a connect sum of two lens spaces each of order 3 . This cannot be contained a lens space, however, since lens spaces are prime.

Lemma 6.10. Up to homeomorphism, each lens space $L(9 k-3,3 k-2)$, $k \in \mathbb{Z}$, contains exactly one order 3 OPT-knot, $K_{k}^{3}$. Every order 3 OPT-knot is one of these.

Proof. Observe that the lemma holds for order 3 torus knots by Lemma 3.1. By Lemmas 6.4 and 6.6, a non-torus knot of order 3 with a properly embedded once-punctured torus in its exterior must have $G_{S}$ appear as in figure 18(b). As shown, $G_{S}$ contains a single Scharlemann cycle of length $3 \sigma$, which bounds two faces $f_{a}$ and $f_{b}$ of colors amber and blue, respectively.

Lemma 6.9 together with Lemma 5.1 implies that $G_{T}$ lies in an essential annulus. With labelings of the edges and corners of $f_{a}$ as in figure 10, $G_{S}$ and $G_{T}$ may be assumed to be as shown in figure 20. Since handlebodies are irreducible, these labelings then dictate the positions of the disks $f_{a}$ and $f_{b}$ in the genus two handlebodies $\left(V_{a}-H_{1,2}\right) \cup \partial_{v} H_{1,2}$ and $\left(V_{b}-H_{2,1}\right) \cup \partial_{v} H_{2,1}$, respectively. A priori the position of $\partial f_{a}$ is determined up to Dehn twists along the core curve $h_{a}$ of $\partial_{v} H_{1,2}$. But since $h_{a}$ is dual to a compressing disk of the handle body and $\partial f_{a}$ intersects $h_{a}$ algebraically (and geometrically) 3

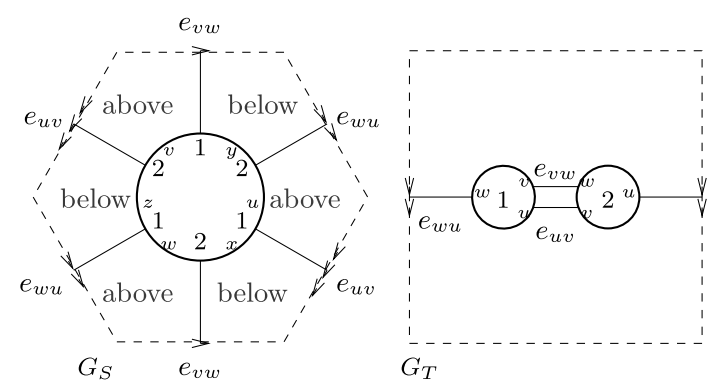

Figure 20: $G_{S}$ of figure 18(b) and $G_{T}$ with labelings of edges and corners. 


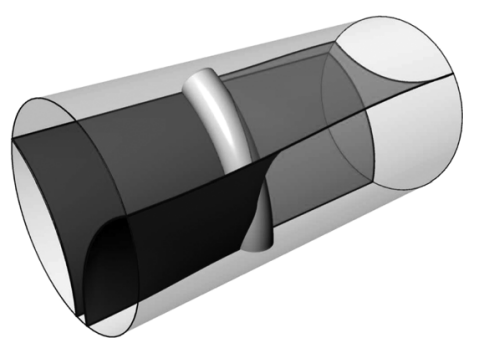

Figure 21: Joining the front to the back with a $2 \pi / 3$ rotation forms the trigon face $f_{i}$ of a length 3 Scharlemann cycle with $K_{i}$ in the solid torus $V_{i}$ for each $i=a, b$.

times, there is at most one configuration for which $\partial f_{a}$ bounds a disk. Such a configuration is depicted in figure 21. The same argument holds for $f_{b}$.

Gluing $V_{a}$ and $V_{b}$ together along $\hat{T}$ so that the edges of $f_{a}$ and $f_{b}$ meet correctly on $G_{T}$ reconstitutes our lens space, our knot $K$ and once-punctured torus $S$. Observe that since $S \cup K$ is disjoint from an essential annulus of $\hat{T}$, there is a one-parameter family of possible lens spaces formed by Dehn twists along this annulus of the gluing map. To determine what lens spaces are thusly produced, we examine how the amber and blue meridians are situated on $\hat{T}$ with respect to one another.

With the given labelings, Lemma 5.4 implies that the amber and blue meridians, $m_{a}$ and $m_{b}$, respectively, lie on $\hat{T}$ with respect to $G_{T}$ as shown in figure 22 each up to Dehn twists along an essential simple closed curve $C$ on $\hat{T}$ that is disjoint from $G_{T}$. Since both meridians are determined up to Dehn twists along $C$, we may fix $m_{b}$ and twist $m_{a}$. Let $\tau_{C}\left(m_{a}\right)$ denote a single Dehn twist of $m_{a}$ along $C$ to the right.

In figure 22, orient $m_{b}$ upwards and rightwards. Let $l$ be a vertical curve oriented upwards. Then $\left\{\left[m_{b}\right],[l]\right\}$ forms a basis for $H_{1}(\hat{T})$. Orient $m_{a}$ downwards and rightwards; orient $C$ rightwards. Then $\left[m_{a}\right]=\left[m_{b}\right]-6[l]$ and $[C]=\left[m_{b}\right]-3[l]$. Since $\Delta\left(m_{a}, C\right)=3,\left[\tau_{C}^{k}\left(m_{a}\right)\right]=\left[m_{a}\right]+3 k[C]=(1+$ $3 k)\left[m_{b}\right]-(6+9 k)[l]$. Therefore, the lens spaces that may be obtained from this construction with $G_{T}$ as shown are $L(-(6+9 k), 1+3 k)$ for $k \in \mathbb{Z}$. We make the reparameterization $k \mapsto k-1$ and apply homeomorphisms to obtain $L(9 k-3,3 k-2)$. Then let $K_{k}^{3}$ be the knot $K$ in $L(9 k-3,3 k-2)$.

Remark 6.3. Since $\Delta\left(m_{a}, C\right)=3$ and $\Delta\left(m_{b}, C\right)=3$, the curve $C$ is a torus knot with exterior $W(-3, \cdot)$; see Lemma 3.1. The order 3 OPT-knots $K_{k}^{3}$ in the lens spaces $L(9 k-3,3 k-2)$ are related by Dehn surgery on $C$. 

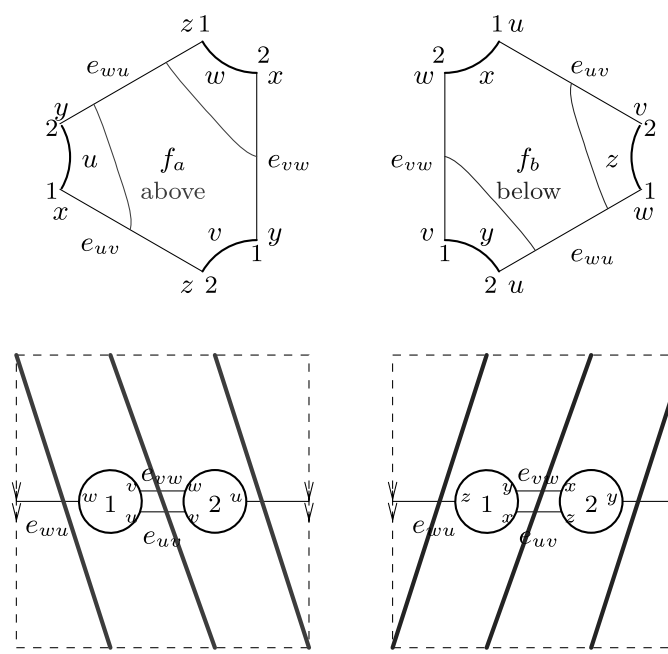

amber meridian

View $G_{T}$ from within $V_{a}$

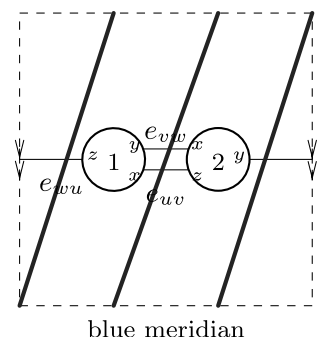

Figure 22: The amber and blue meridional disks intersect the amber and blue faces, $f_{a}$ and $f_{b}$, respectively. The amber and blue meridians then lie on $\hat{T}$ with respect to $G_{T}$ as shown.

Lemma 6.11. The order 3 OPT-knot $K_{k}^{3}$ is the $|3 k-1|$ th grid number 1 knot in the lens space $L(9 k-3,3 k-2)$.

Proof. Write $K_{k}^{3}=K_{a} \cup K_{b}$. Recall $K_{a}$ and $K_{b}$ are each unknotted arcs in $V_{a}$ and $V_{b}$, respectively. By Lemma 5.4 there are meridians $m_{a}$ and $m_{b}$ of $V_{a}$ and $V_{b}$ that bound meridional disks that are disjoint from each $K_{a}$ and $K_{b}$ and intersect $G_{T}$ in a prescribed manner. Attaching $\partial V_{a}$ to $\partial V_{b}$ so that they agree on $G_{T}$ may be done so that $m_{a}$ and $m_{b}$ intersect minimally. Since the vertices of $G_{T}$ mark where $K$ meets $\hat{T}$ and each vertex of $G_{T}$ is in its own parallelogram of the toroidal grid $m_{a} \cup m_{b}, K$ is grid number 1. Since $K$ has order $3, K$ must be the $|r / 3|$ th grid number 1 knot, up to homeomorphism.

Lemma 6.12. The order 3 OPT-knot $K_{k}^{3}$ in the lens space $L(9 k-3,3 k-2)$ is the core of the -3-surgery in $W(-3,-3+1 / k)$.

Proof. By Lemma $2.2 W(-3,-3+1 / k)=N(-2,-2+1 / k, 1)$. Martelli and Petronio identify this as the lens space $L(9 k-3,3 k-2)$ in table A.5 of [2].

Let $K_{k}^{\prime}$ be the core of the 3 -surgery in $W(3,3-1 / k)$. Since the component of $W$ with the surgery coefficient 3 bounds a once-punctured torus 
$S$ that is disjoint from the other component $C$ of $W$, varying the surgery coefficient on $C$ preserves the surface $S$ and the meridian of the core of the 3 -surgery remains distance 3 from $\partial S$. Hence, for each integer $k, K_{k}^{\prime}$ is a knot of order 3 in the lens space $L(9 k-3,3 k-2)$ with the once-punctured torus $S$ properly embedded in its exterior. Therefore, by Lemma 6.10 the knot $K_{k}^{\prime}$ must be the knot $K_{k}^{3}$.

Lemma 6.13. The exterior of $K_{k}^{3}$ in the lens space $L(9 k-3,3 k-2), k \in$ $\mathbb{Z}$, is fibered with fiber $S$ if an only if $k= \pm 1$. Furthermore, $S$ compresses if and only if $k=0$ in which case the knot exterior is a solid torus and $K_{0}^{3}$ is unknotted.

Proof. Since $K_{k}^{3}$ is the core of the -3 -surgery in $W(-3,-3+1 / k)$, its exterior is $W(-3+1 / k, \cdot)$. By Lemma 2.4 this exterior is fibered if and only if $-3+1 / k \in \mathbb{Z}$, i.e., $k= \pm 1$.

If $k=0$ then $K_{0}^{3}$ is the core of three surgery on the unknot in $S^{3}$ and hence its exterior is a solid torus and $S$ compresses. If for some $k \neq 0$ the surface $S$ compresses to a disk $S^{\prime}$ then the neighborhood of $K_{k}^{3} \cup S$ would be a punctured order 3 lens space in a lens space of order other than 3 ; this cannot occur.

Lemma 6.14. The knot $K_{+1}^{3}$ is a type $\{2,4\}$ torus knot in $L(6,1)$. Its exterior is homeomorphic to $W(-2, \cdot)$.

Proof. By Lemma $6.12, K_{+1}^{3}$ is the knot in the lens space $L(6,1)$ that is the core of the -3 -surgery in $W(-3,-2)$. Then by Lemma 3.1 , it is a type $\{2,4\}$ torus knot.

Lemma 6.15. The knot $K_{-1}^{3}$ is a non-torus knot in $L(12,5)$. Its exterior is the once-punctured torus bundle $W(-4, \cdot)$ with monodomy $\phi \cong \tau_{x}^{4} \tau_{y}$.

Proof. Since the exterior of $K_{-1}^{3}$ is homeomorphic to $W(-4, \cdot)$, by Lemma 2.4 the exterior of $K_{-1}^{3}$ is fibered with monodromy $\phi \cong \tau_{x}^{4} \tau_{y}$.

Remark 6.4. Lemmas 6.10 and 6.13 may be proven quite explicitly and graphically using the sutured manifold decomposition (see [35]) of $(E(K), \partial E(K)), K=K_{k}^{3}$. First chop $V_{a} \cap E(K)$ and $V_{b} \cap E(K)$ along the amber and blue trigon faces of $S$ and then reattaching the resulting pieces along the remnants of $\hat{T}$. This leaves one with a sutured manifold that is a genus 2 handlebody $M$ with an annular suture $A$ that divides $\partial M$ into 
two once-punctured tori. In particular $(M, A)$ is the result of the sutured manifold decomposition of $(E(K), \partial E(K))$ along $S$.

When $(M, A)$ admits a complete product disk decomposition, $S$ is the fiber of a fibration of $E(K)$. The monodromy of this fibration may be inferred from this product disk decomposition. One may thusly recover Lemma 6.14 and Lemma 6.15.

We may now continue in this manner to explicitly see the surgery description of $K_{k}^{3}$ of Lemma 6.12. One of the remnants of $\hat{T}$ is an annulus whose core is the curve $C$, and therefore lies in the interior of $M$. In fact one can see that $C$ transversally intersects a compressing disk of $M$ once and is parallel to a curve on $\partial M$ that crosses $A$ twice minimally. Moreover, $(M-N(C), A)$ is homeomorphic to $\left(M^{\prime}-N\left(C^{\prime}\right), A^{\prime}\right)$ where $M^{\prime}$ is the genus 2 handlebody closed neighborhood of a theta graph $C^{\prime} \cup a^{\prime}$ formed from attaching the endpoints of an arc $a^{\prime}$ to distinct points on a circle $C^{\prime}$ and $A^{\prime}$ is an essential annulus on $\partial M^{\prime}$ whose core bounds a disk in $M$ that intersects $C^{\prime} \cup a^{\prime}$ only in $a^{\prime}$. One may now identify $\left(M^{\prime}-N\left(C^{\prime}\right), A^{\prime}\right)$ with the exterior of $W$ chopped along a properly embedded once-punctured torus.

\section{7. $s=2$}

This section is devoted to proving the following theorem.

Theorem 7.1. If $s=2$ then $K$ is a knot in $Y(-2 ;, 1 / k, 1 / \ell)=L(8 k \ell-$ $2,4 k \ell-2 k-1)$ arising as the core of the -2-surgery for some integers $k, \ell \neq$ 0 (and for each pair of integers $k, \ell \neq 0$ there is such a knot). Equivalently, up to homeomorphism $K$ is the $|4 k \ell-2|$ th grid number 1 knot in the lens space $L(8 k \ell-2,4 k \ell-2 k-1)$. Furthermore $E(K)$ is fibered if an only if $|k|=|\ell|=1$ :

- If $k=\ell= \pm 1$ then $K$ is homeomorphic to a torus knot in $L(6,1)$ with monodromy $\phi \cong \tau_{x}^{3} \tau_{y}$.

- If $k=-\ell= \pm 1$ then $K$ is a non-torus knot in $L(10,3)$ with monodromy $\phi \cong \tau_{x}^{5} \tau_{y}$.

Proof. We give an overview of the proof. The first two subsections culminate in Theorem 7.2 showing that if $s=2$ then $t=2$ and $G_{S}$ appears as in figure 23. Then Lemma 7.8 describes the lens spaces which contain knots with such surfaces $S$. Lemma 7.11 gives the surgery description of this family of knots. Lemma 7.12 shows that $E(K)$ is fibered if and only if $|k|=|\ell|=1$ and that $S$ is incompressible if and only if $k \ell \neq 0$. Lemma 7.13 shows that 


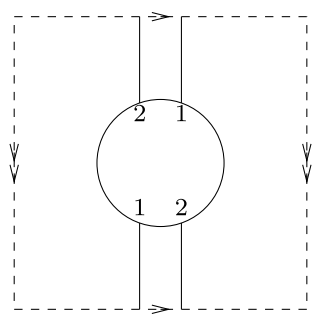

Figure 23: The only viable configuration for $G_{S}$ when $s=2$. (Simple closed curves not included.)

if $k=\ell= \pm 1$ then $K$ is a torus knot. Lemma 3.1 then implies $K \subset L(6,1)$ and has fibered exterior with monodromy $\phi \cong \tau_{x}^{3} \tau_{y}$. Lemma 7.14 shows that if $k=-\ell= \pm 1$ then $K$ has fibered exterior with monodromy $\phi \cong \tau_{x}^{5} \tau_{y}$.

If $s=2$ then Theorem 1.1 of [33] give no apriori bounds on $t$. Nevertheless, we will explicitly conclude that here too, $t=2$.

When $s=2$, the boundary of $S$ runs twice longitudinally along $\partial N(K)$. Within $N(K)$ there is a Möbius band $P$ with the same boundary as $S$. Furthermore we may take $K$ to be the core curve of $P$. Then $S \cup P$ is homeomorphic to $\hat{S} \# \hat{P}$ (where $\hat{P} \cong \mathbb{R} P^{2}$ thought of as abstractly capping off $P$ with a disk). This is a closed non-orientable surface of Euler characteristic -1 , homeomorphic to $\#{ }_{3} \mathbb{R} P^{2}$, and also known as Dyck's surface. Indeed for every Dyck's surface $R$ embedded in the lens space $X$ and orientation reversing simple closed curve $J \subset R$ such that $R-N(J)$ is a once-punctured torus incompressible in $E(J)$, we have an OPT-knot $J$ with an incompressible once-punctured torus Seifert surface $R-N(J)$.

Lemma 7.1. There is a unique isotopy class of simple closed curves $J$ in Dyck's surface $R$ such that $J$ is orientation reversing and $R-N(J)$ is a once-punctured torus.

Proof. We leave this as a fun exercise.

\subsection{When $s=2$ and $r=2$}

Lemma 7.2. An OPT-knot in $L(2,1)$ of order 2 is unknotted.

Proof. Let $K$ be a knot of order 2 in $L(2,1)$ with a once-punctured torus $S$ properly embedded in its exterior. Extend $S$ radially through $N(K)$ to form the Dyck's surface $R$ embedded in $L(2,1)$. 
Identify $V_{a}$ with $S^{1} \times D^{2}$ and $V_{b}$ with $D^{2} \times S^{1}$. We proceed in the vein of Bredon-Wood [36]. Isotop $R$ to intersect $S^{1} \times\{0\}$ transversally. $R$ must intersect $S^{1} \times\{0\}$ an odd number of times, $d$. Re-envision $\hat{T}$ as the boundary of a small tubular neighborhood of this circle and adjust $V_{a}$ and $V_{b}$ accordingly. Then $R$ intersects $V_{a}$ in $d$ meridional disks. The boundary of $R \cap V_{b}$ is then $d$ parallel curves of slope $1 / 2$ on $\partial V_{b}$. We will now isotop $R$ to make $d=1$.

Isotop $R$ to meet the meridional disk $D=D^{2} \times\{1\}$ of $V_{b}$ transversally. Then $R \cap D$ consists of $d$ properly embedded arcs and a collection of simple closed curves. Disregarding simple closed curves, if $d>1$ then an outermost arc of $R \cap D$ on $D$ cuts off a unique disk $E_{b}$ that is disjoint from the other arcs. The two curves on $\partial V_{b}$ that $E_{b}$ meets each bound meridional disks of $V_{a}$ so that there is a product interval $I \times D^{2}$ between them that is disjoint from the rest of $R$ and $E_{b} \cap I \times D^{2}$ is a spanning arc of the annulus $I \times \partial D^{2}$. Let $E_{a}$ be a radial extension of this spanning arc to the arc $I \times\{0\}$. Use $E=E_{a} \cup E_{b}$ to isotop a neighborhood of $E$ in $R$ (including any simple closed curves) to the "other side" of $S^{1} \times\{0\} \subset V_{a}$ by pushing from $R \cap$ $\partial E$ through $E$ to just past $\partial E-R$. This reduces the number of times $R$ intersects $S^{1} \times\{0\}$ by 2 . Rechoose $\hat{T}$ to be the boundary of (a yet smaller) tubular neighborhood of $S^{1} \times\{0\}$ and adjust $V_{a}$ and $V_{b}$ accordingly. Then $R$ intersects $V_{a}$ in $d-2$ meridional disks. Repeat this process until $d=1$.

Now that $d=1, R \cap V_{b}$ may be seen to be a properly embedded Möbius band $P$ with a one-handle attached. We may isotop the feet of this onehandle into a small disk $\Delta$ in $P$ such that both feet are attached to the same side of $\Delta$. Let $J$ be a core curve of $P$ that is disjoint from $\Delta$. Then $J$ is an orientation reversing curve in $R$ and $R-N(J)$ is a once-punctured torus. By Lemma 7.1 $J$ is the unique simple closed curve on $R$ up to isotopy with these two properties. Therefore $J$ is isotopic on $R$ to $K$. One now observes that $J$, and hence $K$, is isotopic to the core of $V_{b}$. Therefore, the exterior of $K$ is a solid torus and $K$ is unknotted. (Furthermore the co-core of the one-handle is a compressing disk for $R$ disjoint from $J$ thereby giving a compression of $S$.)

\subsection{When $s=2$ and $r>2$}

In light of Lemma 7.2, we assume $r>2$. Since $r \neq 2$, Lemmas 5.5 and 5.6 imply that the edges of a Scharlemann cycle of length two of $G_{S}$ must lie in an essential annulus on $G_{T}$.

Theorem 7.2. If $s=2$ then $t=2$ and $G_{S}$ appears as in figure 23. 


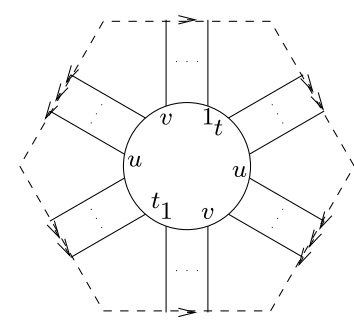

(a)

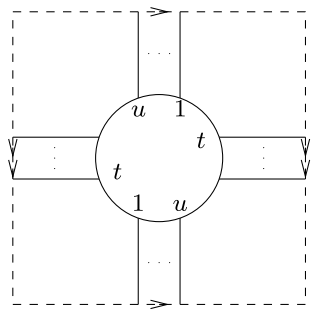

(b)

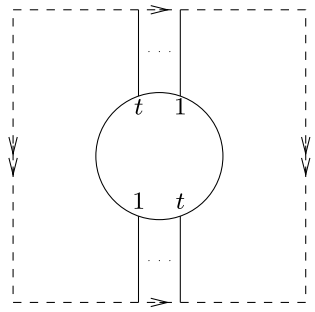

(c)

Figure 24: When $s=2$ and $G_{S}$ has three, two or one edge classes.

Proof. Assume $t>2$. Around the vertex of $G_{S}$ the labels $\{1, \ldots, t\}$ each appear twice. By relabeling if necessary, $G_{S}$ appears as in figure $24(\mathrm{a})$, (b) or (c) according to whether $G_{S}$ has three, two or one edge classes respectively. Below, Lemmas 7.3 and 7.4 show that $G_{S}$ must have just one edge class and Lemma 7.5 shows that $t$ cannot be greater than 2 . The configuration of $G_{S}$ is then forced.

Lemma 7.3. If $s=2$ then $G_{S}$ cannot have three edge classes.

Proof. Assume $G_{S}$ has three edge classes. Each edge class of $G_{S}$ must have at least two edges. By the symmetry of the labelings around $\partial S$, if an edge class had just one edge, it would violate the Parity rule. Now since each edge class has at least two edges then, as evident from figure $24(\mathrm{a}), G_{S}$ has three Scharlemann cycles of length two with mutually disjoint label pairs. By Lemma 5.3 this cannot occur.

Lemma 7.4. If $s=2$ then $G_{S}$ cannot have two edge classes.

Proof. Assume $G_{S}$ has two edge classes as in figure 24(b). Each edge class of $G_{S}$ must have at least two edges. If not then $G_{S}$ appears up to relabeling as in figure $25(\mathrm{a})$ or (b) according to whether just one or two edge classes have just one edge, respectively. Both of these situations fail to satisfy the Parity rule.

Then as evident from figure $24(\mathrm{~b}), G_{S}$ has two Scharlemann cycles of length two, $\sigma$ and $\tau$, with disjoint label pairs. By Lemma 5.3 the faces of $\sigma$ and $\tau$ must have different colors.

There is a tetragon face $f$ of $G_{S}$. We claim that Int $f \cap \hat{T}$ does not contain any curves that are essential on $\hat{T}$.

If not then Int $f \cap \hat{T}$ contains a curve that is essential on $\hat{T}$. After any necessary disk exchanges, there is an innermost disk $D$ on $f$ bounded by an 


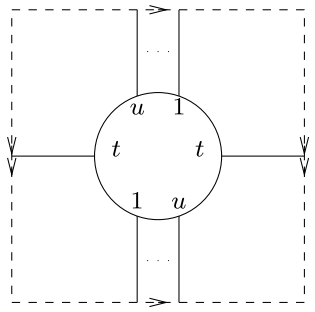

(a)

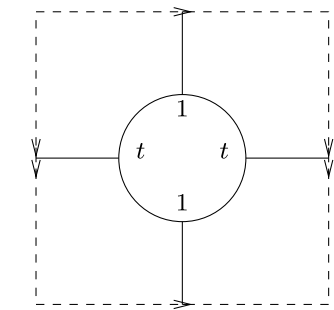

(b)

Figure 25: When $s=2, G_{S}$ has two edge classes, and some edge class has just one edge.

essential curve of Int $f \cap \hat{T}$. One of the Scharlemann cycles, say $\sigma$, has the same color as $D$. Joining the corners of $\sigma$ along $K$ forms a Möbius band whose boundary is disjoint and parallel to $\partial D$ on $\hat{T}$. Then with the annulus of parallelism, the Möbius band and $D$ form a projective plane in a solid torus. This cannot occur.

Given the claim, by disk exchanges we may arrange that Int $f \cap \hat{T}=\emptyset$. Opposite corners of $f$ have the same two labels, either $\{t, 1\}$ or $\{u, u+1\}$. Extend the corners of $f$ radially to the cores of $H_{t, 1}$ and $H_{u, u+1}$ to form the twice punctured projective plane $f^{\prime}$. Since the opposite edges of $f$ each bound (extended) Scharlemann cycles in $G_{S}$, the boundary components of $f^{\prime}$ lie in essential annuli and are therefore parallel. Let $A$ be this annulus of parallelism. Then $f^{\prime} \cup A \cong \mathbb{R} P^{2} \# T^{2}$ is a closed non-orientable surface in a solid torus. This cannot occur.

Lemma 7.5. If $s=2$ and $t>2$ then $G_{S}$ cannot have one edge class.

Proof. Assume $G_{S}$ has one edge class. Then as evident from figure 24(c), $G_{S}$ has an extended Scharlemann cycle of length two, $\sigma$, that contains all of the labels. (Note that this edge class cannot have just one edge.) Let $g$ be the bigon face bounded by the Scharlemann cycle in $\sigma$.

Since $\sigma$ pairs edges of $G_{T}$ so that the pairs lie in essential annuli on $\hat{T}$, no component of $G_{T}$ lies in a disk on $\hat{T}$. Therefore we may perform disk exchanges to eliminate simple closed curves of $S \cap T$ that are trivial on $\hat{T}$. Then the bigons of the extended Scharlemann cycle give rise to a Möbius band and a collection of annuli by extending their corners radially to the core of $H_{1, t}=H_{1,2} \cup \cdots \cup H_{t-1, t}$. The bigon $g$ gives a central Möbius band $A_{0}$. Stepping uniformly away from $g$, the $i$ th pair of bigons forms an annulus $A_{i}$ which shares a boundary component with $A_{i-1}$ for $i=1, \ldots, t / 2$. Hence, we form the long Möbius band $A=A_{0} \cup A_{1} \cup \ldots A_{t / 2}$. Since $K_{t, 1}=H_{t, 1} \cap K$ is 
the only arc of $K-\hat{T}$ not contained in $A$, we may use $A$ to thin $K$ unless $t=2$. See Section 4 of [33].

\subsection{When $G_{S}$ is as in figure 23}

Lemma 7.6. If $s=2$ then $S \cap T$ contains no simple closed curves.

Proof. By Theorem 7.2, $G_{S}$ appears as in figure 23. Let $g$ be the disk face bounded by the Scharlemann cycle. Let $f$ be the annulus face.

Since $r \neq 2$ (Lemma 7.2) the two edges of $G_{T}$ lie in an essential annulus (Lemmas 5.5 and 5.6). By disk exchanges we may assume any simple closed curve of $S \cap T$ is essential on $\hat{T}$. Since lens spaces are prime and $K$ is not contained in a ball, these disk exchanges may be realized by isotopies of $S$ with support in the exterior of $K$.

If $S \cap T$ contains a simple closed curve that bounds a disk on $S$, then assume $D$ is an innermost such disk. By Lemma 5.6 this disk must lie in the annulus face $f$ of $G_{S}$. Form a Möbius band $A_{0}$ by extending the corners of $g$ radially to the core of $H_{1,2}$. Then since $\partial A_{0}$ and $\partial D$ are disjoint essential curves on $\hat{T}$, they are parallel. Let $B$ be an annulus of parallelism between these curves on $\hat{T}$. Then $N\left(B \cup A_{0} \cup D\right)$ is a punctured $L(2,1)$. Since $r \neq 2$, this is a contradiction. Hence any simple closed curve of $S \cap T$ is essential on $S$. Such curves are all contained in the annulus face $f$ of $G_{S}$, and there must be an even number of them.

We isotop $S$ to reduce the number of these curves. Include all the simple closed curves of $S \cap T$ in $G_{S}$ and color the faces so that $g$ (and the corresponding Möbius band $A_{0}$ ) is colored blue. Then every blue annulus is disjoint from $A_{0}$ and may be regarded as the horizontal boundary of a twisted $I$ bundle over $A_{0}$ in $V_{b}$. Moreover, each blue annulus is boundary-parallel into an annulus of $\hat{T}$ through the solid torus complement of its corresponding twisted $I$ bundle in $V_{b}$. Isotop an outermost blue annulus onto $\hat{T}$ and then push it slightly into $V_{a}$. This reduces the number of simple closed curves of $S \cap T$ by two.

Therefore, if $S \cap T$ contains a simple closed curve then $|S \cap T|$ is not minimized. This is a contradiction to our minimality assumption on $|S \cap T|$. Hence $S \cap T$ contains no simple closed curves.

Let us abbreviate "once-punctured Klein bottle" as OPK.

Lemma 7.7. Up to homeomorphism, there is a one-parameter family of properly embedded, incompressible OPK in a solid torus. 

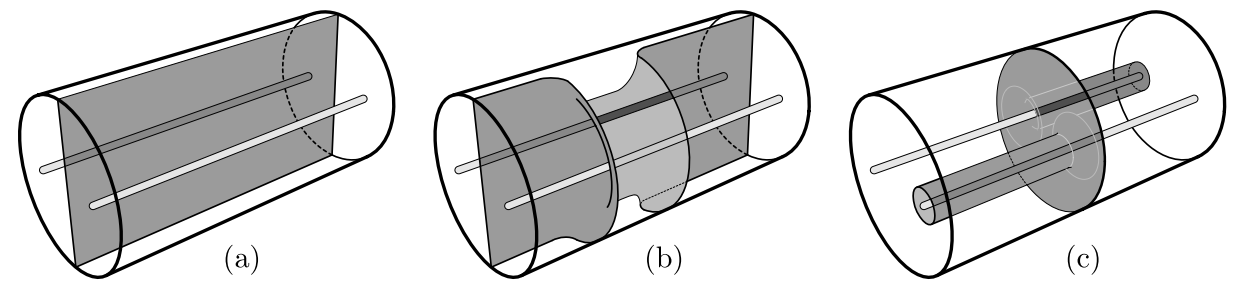

Figure 26: In each, glue the front to the back with a $\pi$ twist to form the solid torus $V_{a}$ containing the $(2,1)$-curve $C_{a}$. The surface in (a) is the Möbius band $F^{\prime}$. The surface in (b) and (c) is the OPK $F$.

Let $C_{a}$ be a $(2,1)$-torus knot on a concentric torus in $V_{a}$ framed by its parallelism into $\partial V_{a}$. Given a meridional disk of $V_{a}$ that $C_{a}$ pierces twice, let $F$ be the OPK that results from tubing the disk to itself along one half of $C_{a}$. A properly embedded, incompressible OPK in a solid torus is homeomorphic to $F \subset V_{a}$ with some $\frac{1}{n}$ surgery on $C_{a}, n \neq 0$. The solid torus $V_{a}$ with $F$ and $C_{a}$ is shown in figure $26(c)$.

Proof. An incompressible OPK $F$ properly embedded in a solid torus $V_{a}$ is $\partial$-compressible. Performing such a $\partial$-compression yields either an annulus or a Möbius band $F^{\prime}$. Undoing the $\partial$-compression is a $\partial$-tubing along an arc in $\partial V_{a}$ with its endpoints on $\partial F^{\prime}$.

Since a properly embedded annulus in a solid torus is separating, it cannot be $\partial$-tubed to form an OPK. Thus, $F^{\prime}$ must a Möbius band. Up to homeomorphism, we may view $F^{\prime} \subset V_{a}$ as in figure 26(a). An arc along which a $\partial$-tubing of $F^{\prime}$ produces an OPK is a spanning arc of the annulus $\partial V_{a}-$ $\partial F^{\prime}$. Such arcs are related by Dehn twists along the core of the annulus. Such Dehn twists may be realized by Dehn surgeries along a push-off $C_{a}$ of the core of the annulus into $V_{a}$. A $\partial$-tubing along one particular arc produces $F$ with $C_{a}$ as shown in figure 26(b), which is isotopic to figure 26(c).

Remark 7.1. Note that a properly embedded OPK in a solid torus is compressible only if its boundary is meridional. Hence, such an OPK in a solid torus must be homeomorphic to $F$ in $V_{a}$ (with the trivial surgery on $C_{a}$ ) as shown in figure $26(\mathrm{c})$.

Lemma 7.8. Up to homeomorphism, each lens space $L(8 k \ell-2,4 k \ell-2 k-$ $1), k, \ell \in \mathbb{Z}$, contains exactly one order 2 OPT-knot $K_{k, \ell}^{2}$. Every order 2 OPT-knot is one of these. 
Remark 7.2. Observe that $L(8 k \ell-2,4 k \ell-2 k-1)$ and $L(8 k \ell-2,4 k \ell-$ $2 \ell-1)$ are orientation preserving homeomorphic.

Remark 7.3. By Corollary 6.4 of [36] and the proof of their Theorem 6.1, a curve of slope $(2 j, q)$ on a solid torus bounds an OPK if and only if $j$ is even and $q \equiv \pm(j-1) \bmod 2 j$. One may then show that attaching a solid torus containing a Möbius band so that a Dyck's surface is formed from the Möbius band and the OPK in the first solid torus produces lens spaces homeomorphic to $L(8 k \ell-2,4 k \ell-2 k-1)$ for $k, \ell \in \mathbb{Z}$. In the proof below we observe this by realizing these lens spaces as surgeries on a pair of torus knots (at different heights) disjoint from a (compressible) Dyck's surface in $L(2,1)$.

Proof. By Theorem 7.2, a non-torus OPT-knot of order 2 must have $G_{S}$ appear as in figure 23 . As shown, $G_{S}$ contains a single Scharlemann cycle of length 2 which bounds an annulus face $f_{a}$ and a bigon face $f_{b}$ of colors amber and blue, respectively.

We label the edges and corners of $f_{a}$ and $f_{b}$ so that $G_{S}$ and $G_{T}$ may be assumed to be as shown in figure 27 .

By extending the corners of $f_{a}$ radially to the core of $H_{1,2}$ we obtain an OPK $\bar{f}_{a}$ properly embedded in $V_{a}$. By Lemma $7.7, \bar{f}_{a}$ appears in $V_{a}$ as in figure $26(\mathrm{c})$ up to $\frac{1}{k}$ surgery on $C_{a}$ and homeomorphisms, and note $\Delta\left(\partial \bar{f}_{a}, m_{a}\right)=4 k$. Similarly $f_{b}$ may be extended to a Möbius band $\bar{f}_{b}$ properly embedded in $V_{b}$ and $\Delta\left(\partial \bar{f}_{b}, m_{b}\right)=2$.

Together $\bar{f}_{a}$ and $\bar{f}_{b}$ form a Dyck's surface $R$ embedded in the lens space and the properly embedded $\operatorname{arcs} K_{a} \subset \bar{f}_{a}$ and $K_{b} \subset \bar{f}_{b}$ form the knot $K$. Lemma 7.1 shows the embedding of $K$ on $R$ is unique up to isotopy. Since $K_{b}$ is necessarily a spanning arc of $\bar{f}_{b}$, the position of $K_{a}$ on $\bar{f}_{a}$ is then determined. Up to homeomorphism and up to surgery on $C_{a}$, these are in the solid tori $V_{a}$ and $V_{b}$ as shown in figure 28(a) and (b). Observe that $K_{a}$
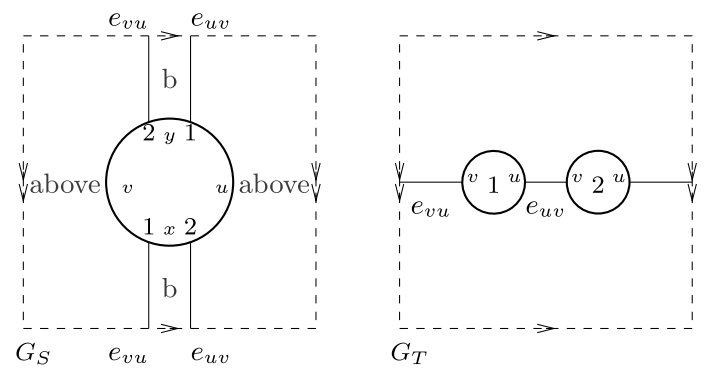

Figure 27: $G_{S}$ of figure 23 and $G_{T}$ with labelings of edges and corners. 

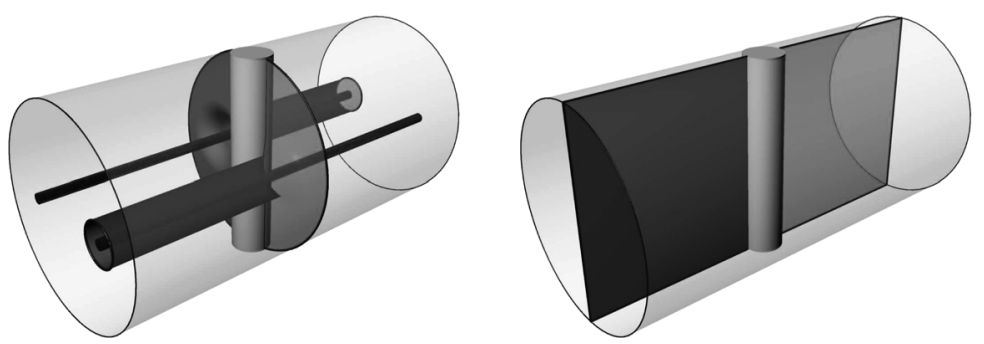

Figure 28: (a) Joining the front to the back with a $\pi$ rotation forms the annulus $f_{a}$ with $K_{a}$ in the solid torus $V_{a}$ up to $1 / k$ surgery on $C_{a}$. (b) Joining the front to the back with a $\pi$ rotation forms the bigon face $f_{b}$ of a length 2 Scharlemann cycle with $K_{b}$ in the solid torus $V_{b}$.

and $K_{b}$ are disjoint from meridional disks of $V_{a}$ and $V_{b}$ bounded by $m_{a}$ and $m_{b}$ respectively.

Gluing $V_{a}$ and $V_{b}$ together along $\hat{T}$ so that the edges of $f_{a}$ and $f_{b}$ meet correctly on $G_{T}$ reconstitutes our lens space, our knot $K$, and oncepunctured torus $S$. (On $\hat{T}$ we have $\partial \bar{f}_{a}=\partial \bar{f}_{b}$ equaling $G_{T}$ with the vertices contracted to a point.) Since $S \cup K=R$ is disjoint from an essential annulus of $\hat{T}$, there is a 1-parameter family of possible lens spaces formed by Dehn twists along the core $C_{b}$ of this annulus of the gluing map (which we may view as $1 / \ell$ surgeries on $C_{b}$, framed by $\hat{T}$ ), in addition to the 1 -parameter family obtained from the $1 / k$ surgeries on $C_{a}$. To determine what lens spaces may thusly be produced, we examine how the amber and blue meridians may be situated on $\hat{T}$ with respect to one another.

Let us begin with $k=0$ for the surgery on $C_{a}$ (even though $S$ is compressible there) so that $\partial \bar{f}_{a}$ and $C_{b}$ on $\hat{T}$ are both parallel to the meridian $m_{a}$. Since $\partial \bar{f}_{a}=\partial \bar{f}_{b}$ and $\Delta\left(\partial \bar{f}_{b}, m_{b}\right)=2$, we may choose the initial gluing of $V_{a}$ to $V_{b}$ so that $m_{b}$ is parallel to $C_{a}$ forming $L(2,1)$. Hence, up to homeomorphism, for a lens space containing an order 2 OPT-knot, $m_{a}$ and $m_{b}$ lie on $\hat{T}$ with respect to $G_{T}$ as shown in figure 29 (a) with, say, $m_{a}$ up to Dehn twists along $C_{a}$ then $C_{b}$. As such, the $1 / k$ and $1 / \ell$ surgeries on $C_{a}$ and $C_{b}$ effect $k$ and $\ell$ right-handed Dehn twists.

Let $\tau_{C}^{n}(\gamma)$ denote a $n$ Dehn twists of $\gamma$ along a curve $C$ to the right if $n>0$ and to the left if $n<0$. With $C$ and $\gamma$ as oriented curves on $\hat{T}$, then $\left[\tau_{C}(\gamma)\right]=[\gamma]+(C \cdot \gamma)[\gamma] \in H_{1}(\hat{T})$ where $C \cdot \gamma$ is the oriented algebraic intersection number.

In figure $29(\mathrm{~b})$, orient $m_{b}$ rightwards and let $l$ be a vertical curve oriented upwards. Then $\left\{\left[m_{b}\right],[l]\right\}$ forms a basis for $H_{1}(\hat{T})$. Orient $m_{a}, C_{b}$, and $C_{a}$ towards the right. Then $\left[m_{a}\right]=\left[C_{b}\right]=\left[m_{b}\right]+2[l]$ and $\left[C_{a}\right]=\left[m_{b}\right]$. Since $C_{a}$. 


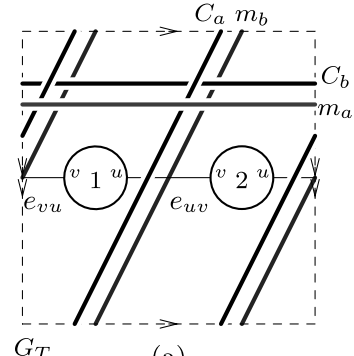

(a)

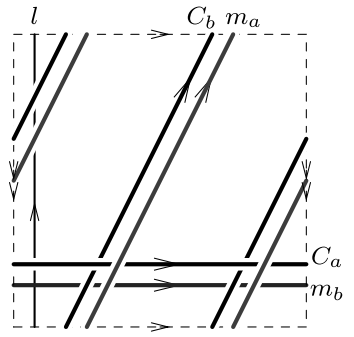

(b)

Figure 29: (a) The amber and blue meridians then lie on $\hat{T}$ with respect to $G_{T}$ as shown up to Dehn twists along $C_{a}$ and $C_{b}$. (b) Redrawn on $\hat{T}$ with basis $\left\{\left[m_{b}\right],[l]\right\}$.

$m_{a}=2$,

$$
\left[\tau_{C_{a}}^{k}\left(m_{a}\right)\right]=\left[m_{a}\right]+2 k\left[C_{a}\right]=(1+2 k)\left[m_{b}\right]+2[l] .
$$

Then $C_{b} \cdot \tau_{C_{a}}^{k}\left(m_{a}\right)=-4 k$ so that

$$
\left[\tau_{C_{b}}^{\ell}\left(\tau_{C_{a}}^{k}\left(m_{a}\right)\right)\right]=\left[\tau_{C_{a}}^{k}\left(m_{a}\right)\right]-4 k \ell\left[C_{b}\right]=(1+2 k-4 k \ell)\left[m_{b}\right]+(-8 k \ell+2)[l] .
$$

Therefore, the lens spaces that may be obtained from this construction are $L(8 k \ell-2,4 k \ell-2 k-1)$. Write $K_{k, \ell}^{2}$ to denote our resulting order 2 -OPT knot $K$ in these lens spaces.

Lemma 7.9. The order 2 OPT-knot $K_{k, \ell}^{2}$ is the $|4 k \ell-1|$ th grid number 1 knot in the lens space $L(8 k \ell-2,4 k \ell-2 k-1)$.

Proof. In $L(2,1)$, when $k=0$ or $\ell=0$, there are meridians $m_{a}$ and $m_{b}$ for $V_{a}$ and $V_{b}$ bounding meridional disks $D_{a}$ and $D_{b}$ disjoint from the knot $K=K_{0,0}^{2}$ such that $m_{a}$ and $m_{b}$ intersect minimally (twice) on $\hat{T}$. Indeed $N\left(D_{a} \cup D_{b}\right)$ is the exterior of this knot. Thus $K_{0,0}^{2}\left(=K_{0, \ell}^{2}=K_{k, 0}^{2}\right)$ is the grid number 1 knot in $L(2,1)$.

View the surgeries on $C_{a}$ and $C_{b}$ as changing the attaching of $V_{a}$ and $V_{b}$ by Dehn twists of $m_{a}$ and $m_{b}$, respectively, on $\hat{T}$. Once projected to $\hat{T}$, $C_{a}$ equals $m_{b}$ and $C_{b}$ equals $m_{a}$ in the twice-punctured torus $\hat{T}-N(K)=$ $N\left(m_{a} \cup m_{b}\right)$. Then in $\hat{T}-N(K), \tau_{C_{a}}^{k}\left(m_{a}\right)$ intersects $m_{b}$ algebraically and geometrically the same number of times, and similarly so does $\tau_{C_{a}}^{k}\left(m_{a}\right)$ and $\tau_{C_{b}}^{\ell}\left(m_{b}\right)$. Thus, after the surgeries on $C_{a}$ and $C_{b}$ there are meridional disks for the new solid tori $V_{a}$ and $V_{b}$ that are disjoint from $K=K_{k, \ell}^{2}$ and whose boundaries intersect minimally on $\hat{T}$. Because $K$ continues to be one-bridge with respect to $\hat{T}, K$ has grid number 1 . 
Since $K$ has order $2, K$ must be the $|r / 2|$ th grid number 1 knot. Here, $|r|=|8 k \ell-2|$.

Lemma 7.10. The exterior of the link $K \cup C_{a} \cup C_{b}$ in $L(2,1)$ is homeomorphic to the exterior of the link $Y$, the "twisted" two-chain and axis in figure 1. The -2 surgery on the axis of $Y$ yields $L(2,1)$, sends the two-chain to $C_{a} \cup C_{b}$ preserving their framings, and $K$ arises as the core of the surgery.

Proof. Thicken $V_{a}$ and $V_{b}$ so that they overlap on a $T^{2} \times I$ and so that the curve $C_{a}$ is a meridian of $V_{b}$ and the curve $C_{b}$ is a meridian of $V_{a}$. As such, they bound meridional disks $D_{b}$ and $D_{a}$, respectively, that transversally intersect each other twice with clasp singularities. The exterior of $K$ is the closure of $N\left(D_{a} \cup D_{b}\right)$ as indicated by figure 29 (a) which is a solid torus (cf. Lemma 7.2). This solid torus has an embedding in $S^{3}$ as the exterior of the axis of $Y$, thereby presenting $C_{a} \cup C_{b}$ as the half-twisted two-chain. Figure 30 shows this embedding of the curves $C_{a}$ and $C_{b}$ bounding the disks $D_{b}$ and $D_{a}$. Hence the exteriors of $Y$ and $K \cup C_{a} \cup C_{b}$ are homeomorphic. Moreover, examining figure 29(a) again, one may expand one of the meridians $\partial N(K) \cap$ $\hat{T}$ along $\hat{T}-\left(D_{a} \cup D_{b}\right)$ to lie upon $D_{a} \cup D_{b}$ as the green curve $\gamma$ in figure 30 . Hence, the meridian $\gamma$ of $K$ is sent to the -2 slope on the axis of $Y$. Thus $Y$ has the desired surgery property.

\section{Lemma 7.11.}

- The knot $K_{k, \ell}^{2}$ in the lens space $L(8 k \ell-2,4 k \ell-2 k-1), k, \ell \in \mathbb{Z}$ is the core of the -2-surgery in $Y(-2,1 / k, 1 / \ell)$.

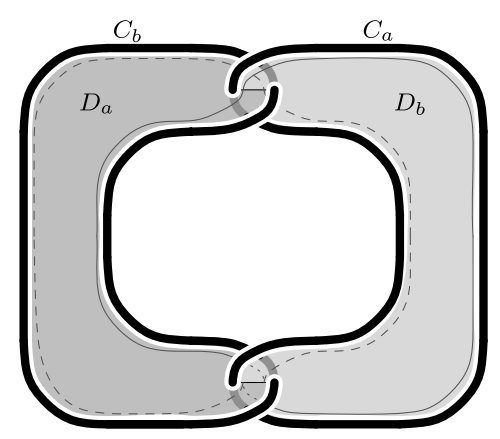

Figure 30: The curves $C_{a}$ and $C_{b}$ bound disks $D_{b}$ and $D_{a}$. In green is a meridian of $K$ (the boundary of vertex 1 in figure 29(a), say) pushed along $\hat{T}$ onto $D_{a} \cup D_{b}$. 
- The knot $K_{k,+1}^{2}$ in the lens space $L(8 k-2,2 k-1)$ is the core of the -2 -surgery in $W(-2,-4+1 / k)$.

- The knot $K_{k,-1}^{2}$ in the lens space $L(8 k+2,6 k+1)$ is the core of the -2 -surgery in the mirror of $W(-2,-4-1 / k)$.

Proof. The first statement follows from the proof of Lemmas 7.8 and 7.10. The second and third are obtained by then applying Lemma 2.2.

Lemma 7.12. The exterior of $K_{k, \ell}^{2}$ in the lens space $L(8 k \ell-2,4 k \ell-2 k-$ $1), k, \ell \in \mathbb{Z}$, is fibered with fiber $S$ if and only if $|k|=|\ell|=1$. Furthermore $S$ compresses if and only if $k=0$ or $\ell=0$ in which case the knot exterior is a solid torus and $K_{k, \ell}^{2}$ is unknotted.

Proof. Since the exterior of $K_{k, \ell}^{2}$ is homeomorphic to $Y(\cdot ; 1 / k, 1 / \ell)$, this follows from Lemma 2.5.

Lemma 7.13. The knot $K_{+1,+1}^{2}$ is a type $\{3,3\}$ torus knot in $L(6,1)$. Its exterior is homeomorphic to $W(-3, \cdot)$. Similarly, the knot $K_{-1,-1}^{2}$ is a type $\{3,3\}$ torus knot in $L(6,5)$ and is homeomorphic to $K_{+1,+1}^{2}$.

Proof. By Lemma 7.11, $K_{+1,+1}^{2}$ is the knot in the lens space $L(6,1)$ that is the core of the -2 -surgery in $W(-2,-3)=W(-3,-2)$. Then by Lemma 3.1, it is a type $\{3,3\}$ torus knot. Lemma 7.11 gives the last statement.

Lemma 7.14. The knot $K_{-1,+1}^{2}$ is a knot in $L(10,3)$ and its exterior is a once-punctured torus bundle with monodomy $\phi=\tau_{x}^{5} \tau_{y}$.

Proof. By Lemma 7.11, $K_{-1,+1}^{2}$ is the knot in the lens space $L(10,3)$ that is, the core of the -2 -surgery in $W(-2,-5)=W(-5,-2)$. Its exterior is then homeomorphic to $W(-5, \cdot)$ which by Lemma 2.4 is fibered with monodromy $\phi \cong \tau_{x}^{5} \tau_{y}$.

Remark 7.4. As in Remark 6.4, but with a bit more work, we may here too use sutured manifolds to obtain explicit proofs of Lemmas 7.8, 7.11, $7.12,7.13$ and 7.14 .

\section{Acknowledgments}

This work was partially supported by NSF Grant DMS-0089927 and NSF Grant DMS-0239600. We thank John Etnyre for some useful comments and suggestions and the referees for their careful readings. 


\section{References}

[1] Kenneth L. Baker, Counting genus one fibered knots in lens spaces, arXiv:math.GT/0510391.

[2] Bruno Martelli and Carlo Petronio, Dehn filling of the "magic" 3-manifold, Comm. Anal. Geom. 14(5) (2006), 969-1026.

[3] Gerhard Burde and Heiner Zieschang, Neuwirthsche Knoten und Flächenabbildungen, Abh. Math. Sem. Univ. Hamburg, 31 (1967), 239-246.

[4] Francisco González-Acuña, Dehn's construction on knots, Bol. Soc. Mat. Mexicana (2) 15 (1970), 58-79.

[5] Kanji Morimoto, Genus one fibered knots in lens spaces, J. Math. Soc. Japan, 41(1) (1989), 81-96.

[6] John A. Baldwin, A note on genus one fibered knots in lens spaces, arXiv:math.GT/0607370.

[7] Andrew J. Casson and Steven A. Bleiler, Automorphisms of surfaces after Nielsen and Thurston, volume 9 of London Mathematical Society Student Texts. Cambridge University Press, Cambridge, 1988.

[8] Kenneth L. Baker, Jesse E. Johnson and Elizabeth A. Klodginski, Tunnel number one, genus one fibered knots, Comm. Ann. Geom. 17(1) (2009), 116.

[9] Ciprian Manolescu, Peter Ozsvath and Sucharit Sarkar, A combinatorial description of knot Floer homology, Ann. of Math. (2) 169(2) (2009), 633-660.

[10] Ciprian Manolescu, Peter Ozsvath, Zoltan Szabo and Dylan Thurston, On combinatorial link Floer homology, Geom. Topol. 11 (2007), 23392412 .

[11] Peter R. Cromwell, Arc presentations of knots and links, in Knot theory (Warsaw, 1995), volume 42 of Banach Center Publ., pp. 57-64. Polish Acad. Sci., Warsaw, 1998.

[12] I. A. Dynnikov, Arc-presentations of links: monotonic simplification, Fund. Math. 190 (2006), 29-76.

[13] J. Elisenda Grigsby, Combinatorial description of knot Floer homology of cyclic branched covers, arXiv:math.GT/0610238. 
[14] Peter Ozsváth and Zoltán Szabó, Knot Floer homology and rational surgeries, arXiv:math.GT/0504404.

[15] Jacob Rasmussen, Lens space surgeries and L-space homology spheres, arXiv:math.GT/0710.2531.

[16] Matthew Hedden, On Floer homology and the Berge conjecture on knots admitting lens space surgeries, Trans. Amer. Math. Soc., 363(2) (2011), 949-968.

[17] Kenneth L. Baker, J. Elisenda Grigsby and Matthew Hedden, Grid diagrams for lens spaces and combinatorial knot Floer homology, Int. Math. Res. Not. IMRN, (2008), no. 10, Art. ID rnm024, 39 pp.

[18] John Berge, Some knots with surgeries yielding lens spaces. Unpublished manuscript.

[19] Peter Ozsváth and Zoltán Szabó, On knot Floer homology and lens space surgeries, Topology, 44(6) (2005), 1281-1300.

[20] John Stallings, On fibering certain 3-manifolds, in Topology of 3-manifolds and related topics (Proc. The Univ. of Georgia Institute, 1961), pp. 95-100. Prentice-Hall, Englewood Cliffs, N.J., 1962.

[21] K. S. Brown, Trees, valuations, and the Bieri-Neumann-Strebel invariant, Invent. Math. 90(3) (1987), 479504.

[22] Cameron McA. Gordon, Small surfaces and Dehn filling, in Proceedings of the Kirbyfest (Berkeley, CA, 1998), vol. 2 of Geom. Topol. Monogr., pp. 177-199 (electronic). Geom. Topol. Publ., Coventry, 1999.

[23] Cameron McA. Gordon and Ying-Qing Wu, Toroidal and annular Dehn fillings, Proc. London Math. Soc. 78(3) (1999), 662-700.

[24] R. Gompf and A. Stipsicz, 4-manifolds and Kirby calculus, vol. 20 of Graduate Studies in Mathematics. American Mathematical Society, Providence, RI, 1999.

[25] Craig D. Hodgson, G. Robert Meyerhoff and Jeffrey R. Weeks, Surgeries on the Whitehead link yield geometrically similar manifolds, in Topology '90 (Columbus, OH, 1990), vol. 1 of Ohio State Univ. Math. Res. Inst. Publ., pp. 195-206. de Gruyter, Berlin, 1992.

[26] Francis Bonahon and Jean-Pierre Otal, Scindements de Heegaard des espaces lenticulaires, Ann. Sci. École Norm. Sup. (4) 16(3) (1984), 451466, 1983. 
[27] Richard A. Litherland, Surgery on knots in solid tori. II, J. London Math. Soc. (2) 22(3) (1980), 559-569.

[28] David Gabai, Foliations and the topology of 3-manifolds III, J. Differential Geom. 26(3) (1987), 479-536.

[29] Yo'av Rieck, Heegaard structures of manifolds in the Dehn filling space, Topology, 39(3) (2000), 619-641.

[30] Cameron McA. Gordon, Combinatorial methods in Dehn surgery, in Lectures at KNOTS '96 (Tokyo), vol. 15 of Ser. Knots Everything, pp. 263-290. World Sci. Publishing, River Edge, NJ, 1997.

[31] Hiroshi Goda and Masakazu Teragaito, Dehn surgeries on knots which yield lens spaces and genera of knots, Math. Proc. Cambridge Philos. Soc. 129(3) (2000), 501-515.

[32] Marc Culler, Cameron McA. Gordon, John Luecke and Peter B. Shalen, Dehn surgery on knots, Bull. Amer. Math. Soc. (N.S.), 13(1) (1985), 43-45.

[33] Kenneth L. Baker, Small genus knots in lens spaces have small bridge number, Algebr. Geom. Topol. 6 (2006), 1519-1621 (electronic).

[34] C. McA. Gordon and J. Luecke, Dehn surgeries on knots creating essential tori I, Comm. Anal. Geom. 3(3-4) (1995), 597-644.

[35] David Gabai, Foliations and the topology of 3-manifolds, J. Differential Geom. 18(3) (1983), 445-503.

[36] Glen E. Bredon and John W. Wood, Non-orientable surfaces in orientable 3-manifolds, Invent. Math. 7 (1969), 83-110.

Department of Mathematics

UNIVERSITY OF MiAMI

Coral Gables, FL 33146

USA

E-mail address: k.baker@math.miami.edu

Received November 6, 2008 
